\title{
Three-point correlators from string amplitudes: mixing and Regge spins
}

\author{
Joseph A. Minahan and Raul Pereira \\ Department of Physics and Astronomy, Uppsala University, \\ Box 516, SE-751 20 Uppsala, Sweden \\ E-mail: joseph.minahan@physics.uu.se, raul.pereira@physics.uu.se
}

AbStRaCT: This paper has two parts. We first compute the leading contribution to the strong-coupling mixing between the Konishi operator and a double-trace operator composed of chiral primaries by using flat-space vertex operators for the string-duals of the operators. We then compute the three-point functions for protected or unprotected scalar operators with higher spin operators on the leading Regge trajectory. Here we see that the nontrivial spatial structures required by conformal invariance arise naturally from the form of the polarization tensors in the vertex operators. We find agreement with recent results extracted from Mellin amplitudes for four-point functions, as well as with earlier supergravity calculations. We also obtain some new results for other combinations of operators.

KEYwORDS: Supersymmetric gauge theory, Superstrings and Heterotic Strings, AdS-CFT Correspondence, Strong Coupling Expansion

ARXIV EPRINT: 1410.4746 


\section{Contents}

1 Introduction 1

2 Review of previous results 3

3 Extremal correlators and operator mixing 5

4 Three-point couplings with spin $\quad 7$

$\begin{array}{lll}4.1 & \text { The operators and corresponding string states } & 7\end{array}$

4.2 The building blocks 9

$\begin{array}{lll}4.3 & \text { Structure constants } & 13\end{array}$

5 Discussion $\quad 17$

$\begin{array}{lr}\text { A Vertex function contractions } & 18\end{array}$

\section{Introduction}

We can now compute the dimensions of single-trace local operators in planar $\mathcal{N}=4$ super Yang-Mills (SYM), at least in principle, because of its underlying integrability [1]. However, to fully solve the theory it is also necessary to know the three-point functions between local operators. There are now a number of important results on this subject at both weak [2-15] and strong coupling [16-27].

In this paper we continue our study of three-point correlators in $\mathcal{N}=4 \mathrm{SYM}$ at strong coupling by using flat-space vertex operators to find the relevant couplings [28, 29]. Here we investigate two issues. The first involves the appearance of poles in extremal three-point amplitudes. This leads to a mixing between single- and double-trace operators which we explicitly compute for a Konishi operator mixing with a double-trace operator composed of two chiral primaries.

The second issue we consider is the three-point functions for scalar operators with a higher spin operator. Using results of Schlotterer for flat-space massive superstring amplitudes [30], we can straightforwardly compute the three-point functions and compare with recent results in [31], where the authors study these correlators by considering Regge amplitudes of four scalar operators. In the limit of large dimensions we find agreement with their results.

In a conformal field theory, the correlator of three local scalar operators must have the form

$$
\left\langle\mathcal{O}_{\Delta_{1}}\left(x_{1}^{\mu}\right) \mathcal{O}_{\Delta_{2}}\left(x_{2}^{\mu}\right) \mathcal{O}_{\Delta_{3}}\left(x_{3}^{\mu}\right)\right\rangle=\frac{\mathcal{C}_{123}}{\left|x_{12}\right|^{\Delta_{1}+\Delta_{2}-\Delta_{3}}\left|x_{23}\right|^{\Delta_{2}+\Delta_{3}-\Delta_{1}}\left|x_{31}\right|^{\Delta_{3}+\Delta_{1}-\Delta_{2}}},
$$


where $\Delta_{i}$ are the operator dimensions. Using Witten diagrams [32], one finds that the structure constant $\mathcal{C}_{123}$ in a four-dimensional CFT has the form [33]

$$
\mathcal{C}_{123}=\frac{\sqrt{\left(\Delta_{1}-1\right)\left(\Delta_{2}-1\right)\left(\Delta_{3}-1\right)}}{2^{5 / 2} \pi} \frac{\Gamma\left(\alpha_{1}\right) \Gamma\left(\alpha_{2}\right) \Gamma\left(\alpha_{3}\right) \Gamma(\Sigma-2)}{\Gamma\left(\Delta_{1}\right) \Gamma\left(\Delta_{2}\right) \Gamma\left(\Delta_{3}\right)} \mathcal{G}_{123},
$$

where

$$
\Sigma=\frac{1}{2}\left(\Delta_{1}+\Delta_{2}+\Delta_{3}\right), \quad \alpha_{i}=\Sigma-\Delta_{i} .
$$

The coupling $\mathcal{G}_{123}$ is given by

$$
\mathcal{G}_{123}=\frac{8 \pi}{g_{\mathrm{c}}^{2} \alpha^{\prime}}\left\langle V_{k_{1}} V_{k_{2}} V_{k_{3}}\right\rangle\left\langle\psi_{J_{1}} \psi_{J_{2}} \psi_{J_{3}}\right\rangle
$$

where $\left\langle\psi_{J_{1}} \psi_{J_{2}} \psi_{J_{3}}\right\rangle$ is an $S^{5}$ overlap integral and $\left\langle V_{k_{1}} V_{k_{2}} V_{k_{3}}\right\rangle$ is the 3-point string amplitude for the string states dual to the operators, with the momenta $k_{i}$ depending on their dimensions and $R$-charges. The closed string coupling and tension are translated through the AdS/CFT dictionary to be $g_{\mathrm{c}}=\pi^{3 / 2} / N$ and $\alpha^{\prime}=1 / \sqrt{\lambda}$, where $\lambda=g_{Y M}^{2} N$ is the 't Hooft coupling.

In supergravity amplitudes the three-point couplings may have derivative terms, but this does not affect our results since our string amplitudes are on-shell and do not distinguish between derivative and non-derivative couplings. As an illustration of this point, the difference between Witten diagrams for the supergravity couplings $\phi_{1} \phi_{2} \phi_{3}$ and $\phi_{1} \partial_{\mu} \phi_{2} \partial^{\mu} \phi_{3}$ is the factor [33]

$$
\Delta_{2} \Delta_{3}+(d-2 \Sigma) \alpha_{1} \approx \frac{1}{2}\left(\Delta_{1}^{2}-\Delta_{2}^{2}-\Delta_{3}^{2}\right)
$$

where the approximation assumes that $\Delta_{i} \gg 1$. But this last factor is precisely $-k_{2} \cdot k_{3}$ where the $k_{i}$ correspond to the momentum along the $\mathrm{AdS}_{5}$ directions in the flat-space limit [28]. Hence one should use the Witten diagram for non-derivative couplings since the derivative terms, if any, will be built into $\mathcal{G}_{123}$ in our analysis. Further evidence that this is correct, at least to leading order, is our ability to reproduce the supergravity results [34] for three-point functions of chiral primaries in [29], and our results we present in section 4 which among other things reproduce the supergravity results in [45].

A prominent feature of (1.1) is the presence of poles for extremal correlators, which occur when one of the $\alpha_{i}$ is zero. This corresponds to the dimension of one operator being equal to the sum of the other two. In the case of three chiral primaries it was shown that the pole is canceled by a zero in the coupling $\mathcal{G}_{123}$, leaving a finite result $[34,35]$. However, if the correlator contains one or more non-primary operators then this might not be the case. In particular, in [29], the coupling for two chiral primaries with opposite $R$-charges and the Konishi operator was explicitly computed, where it was found that

$$
\left\langle V_{C, J} V_{C,-J} V_{K}\right\rangle \approx \frac{g_{\mathrm{c}}^{3}}{16} \alpha^{\prime 2}\left(J+\frac{1}{2} \Delta\right)^{4} .
$$

$V_{C, J}$ is the vertex operator for a chiral primary $\mathcal{O}_{J}(x)$ with $R$-charge $J$ and $V_{K}$ is the vertex operator for the Konishi operator, with dimension $\Delta \approx 2 \lambda^{1 / 4}$. As extremality is approached with $J \rightarrow_{+} \Delta / 2$ it is clear that the coupling in (1.6) remains non-zero and the pole in (1.1) 
survives. This indicates that the Konishi operator mixes with the double-traced $\mathrm{SO}(6)$ singlet in the tensor product of two $J$-symmetric traceless representations, with

$$
\mathcal{O}_{J \bar{J}}(x)=: \mathcal{O}_{J}(x) \mathcal{O}_{-J}(x):
$$

being one of the components that contribute equally to the mixing.

In this paper we compute the mixing for large $J$, and hence the splitting of the dimensions at the extremal point. If two equal dimension operators mix, there will be a log divergence in the two-point function between the operators. Our result at closest approach of the splitting between the two eigenvalues of the mixed operators is

$$
\Delta m \approx \frac{\sqrt{M} J^{3 / 2}}{N}
$$

where $M$ is the dimension of the $J$-symmetric, traceless representation of $\mathrm{SO}(6)$,

In section 2 we review results from $[28,29]$ which are necessary for the analysis in this paper. In section 3 we present the details about the three-point correlators between the Konishi operator and two chiral primaries of opposite $R$-charge. We then show how this leads to the mixing between Konishi and the double-trace operators. In section 4 we consider the three-point correlators between scalar operators dual to supergravity states and states at the first massive level, and at least one higher spin operator dual to a string state along the leading Regge trajectory. We show here that we can match with the results in [31] as long as the scalars also have large $R$-charges such that our flat-space approximations are valid. In section 5 we present our conclusions.

\section{Review of previous results}

In this section we collect some relevant results from [28, 29].

We assume that the operators are short operators such that the sizes of the string-duals are small compared to the $\mathrm{AdS}_{5}$ and $\mathrm{S}^{5}$ radii. We can then approximate the three-point correlator using Witten diagrams [32, 33]. The Witten diagram includes an integration over all possible intersection points, but in the case of large dimension operators the integration is dominated by a saddle point. The location of the saddle point can be determined [28, 36] as well as the contribution of Gaussian fluctuations. The saddle-point location is itself related to the conservation of constants of the motion, and it is these constants that determine the vertex operators that are inserted in the Witten diagram [28].

The classical constants of the motion are expectation values of the different components of the conformal algebra and are determined by the $R$-charges, the spins, the dimensions and by the positions of the operators on the boundary. For example, for the scalar operator $\mathcal{O}_{i}\left(x_{i}\right)$ in a three-point function the conserved charges include

$$
\begin{aligned}
\left\langle P_{i}^{\mu}\right\rangle & \equiv\left\langle\mathcal{O}_{j}\left(x_{j}\right) \mathcal{O}_{k}\left(x_{k}\right) P^{\mu} \mathcal{O}_{i}\left(x_{i}\right)\right\rangle=-2 i\left(\alpha_{k} \frac{x_{i j}^{\mu}}{x_{i j}^{2}}+\alpha_{j} \frac{x_{i k}^{\mu}}{x_{i k}^{2}}\right) \\
\left\langle D_{i}\right\rangle & \equiv\left\langle\mathcal{O}_{j}\left(x_{j}\right) \mathcal{O}_{k}\left(x_{k}\right) D \mathcal{O}_{i}\left(x_{i}\right)\right\rangle=i\left(\Delta_{i}-2 x_{i \mu}\left(\alpha_{k} \frac{x_{i j}^{\mu}}{x_{i j}^{2}}+\alpha_{j} \frac{x_{i k}^{\mu}}{x_{i k}^{2}}\right)\right),
\end{aligned}
$$

where $x_{i j}^{\mu}=x_{i}^{\mu}-x_{j}^{\mu}$. 
The conformal algebra can be written in a manifestly $\mathrm{SO}(2, d)$ covariant way by defining

$$
M_{-1 \mu} \equiv \frac{1}{\sqrt{2}}\left(\kappa P_{\mu}-\kappa^{-1} K_{\mu}\right), \quad M_{d \mu} \equiv \frac{1}{\sqrt{2}}\left(\kappa P_{\mu}+\kappa^{-1} K_{\mu}\right), \quad M_{-1 d} \equiv-D,
$$

with arbitrary $\kappa$, and Casimir $-\frac{1}{2} M_{r s} M^{r s}=-\Delta^{2}, r, s=-1 \ldots d$. Setting $d=4$ and combining with the $\mathrm{SO}(6) R$-symmetry Casimir, we have

$$
-\frac{1}{2} M_{r s} M^{r s}+\frac{1}{2} R_{I J} R^{I J}=-\Delta^{2}+J^{2},
$$

where $R_{I J}$ are the $R$-symmetry generators with $I, J=5, \ldots, 10$.

If $\Delta$ and $J$ are large, then the $\mathrm{SO}(2,4) \times \mathrm{SO}(6)$ algebra effectively reduces to a 10 dimensional Poincaré algebra. Because of the translation symmetry, we are free to shift the intersection point to $x^{\mu}=0$, in which case all $\left\langle M_{\mu \nu}\right\rangle=0$ and the conserved charges $\left\langle K_{i}^{\mu}\right\rangle$ satisfy

$$
\left\langle K_{i}^{\mu}\right\rangle=-\frac{\alpha_{1} \alpha_{2} \alpha_{3} \Sigma}{2 F^{2}} x_{12}^{2} x_{23}^{2} x_{31}^{2}\left\langle P_{i}^{\mu}\right\rangle
$$

where

$$
F=\alpha_{1} \alpha_{2} x_{12}^{2}+\alpha_{2} \alpha_{3} x_{23}^{2}+\alpha_{3} \alpha_{1} x_{13}^{2} .
$$

If we further choose $\kappa$ to be

$$
\kappa=\frac{\sqrt{\alpha_{1} \alpha_{2} \alpha_{3} \Sigma}}{\sqrt{2} F}\left|x_{12}\right|\left|x_{23}\right|\left|x_{31}\right|,
$$

then the only non-zero components in $(2.2)$ are $\left\langle M_{-1 m}\right\rangle, m=0, \ldots, 4$ for all three operators. Assuming $\Delta^{2} \gg 1$, and choosing a basis where the only non-zero $R$-symmetry components are $\left\langle R_{J, 10}\right\rangle$, we can then identify the full 10 -dimensional flat-space momentum as

$$
k^{M}=\left(\left\langle M_{-1 m}\right\rangle,\left\langle R_{J, 10}\right\rangle\right)
$$

which satisfies the on-shell condition

$$
k \cdot k=-\Delta^{2}+J^{2}=-4 n \sqrt{\lambda}
$$

We can expand the algebra to the full superconformal $\operatorname{PSU}(2,2 \mid 4)$, by including the supercharges $Q_{\alpha a}$ and $\widetilde{Q}_{\dot{\alpha}}^{a}$ and the superconformal generators $S_{\alpha}^{a}$ and $\widetilde{S}_{\dot{\alpha} a}$, where $\alpha$ and $\dot{\alpha}$ are space-time spinor indices and raised or lowered $a$ is an $\mathrm{SO}(6)$ spinor index. The $\mathrm{SU}(2,2) \simeq \mathrm{SO}(2,4)$ covariant supergenerators are

$$
\begin{aligned}
Q_{\dot{a} a}^{1} & \equiv\left(\kappa^{1 / 2} Q_{\alpha a}, \kappa^{-1 / 2} \tilde{S}_{\dot{\alpha} a}\right) \\
Q^{2, \dot{a} a} & \equiv\left(\kappa^{-1 / 2} \varepsilon^{\alpha \beta} S_{\beta}^{a}, \kappa^{1 / 2} \varepsilon^{\dot{\alpha} \dot{\beta}} \tilde{Q}_{\dot{\beta}}^{a}\right)
\end{aligned}
$$

where lowered or raised $\dot{a}$ are $\mathrm{SO}(2,4)$ spinor indices. Defining the supercharges

$$
Q_{A}^{\mathrm{L}}=Q_{\dot{a} a}^{1}+\gamma_{\dot{b} \dot{a}}^{-1} \gamma_{b a}^{6} Q^{2, \dot{b} b}, \quad Q_{A}^{\mathrm{R}}=-i\left(Q_{\dot{a} a}^{1}-\gamma_{\dot{b} \dot{a}}^{-1} \gamma_{b a}^{6} Q^{2, \dot{b} b}\right),
$$


then in the flat-space limit these approach the usual 10-dimensional super-Poincaré generators with

$$
\left\{Q_{A}^{\mathrm{L}, \mathrm{R}}, Q_{B}^{\mathrm{L}, \mathrm{R}}\right\}=-2\left(\mathrm{P}_{+} \Gamma^{M} C\right)_{A B} P_{M}, \quad P_{M}=\left(M_{-1 m}, R_{J, 10}\right), \quad\left\{Q_{A}^{\mathrm{L}}, Q_{B}^{\mathrm{R}}\right\}=0,
$$

where $\mathrm{P}_{+}$is the positive-chirality projector.

A special class of operators are primary, which satisfy $\left[S_{\alpha}^{a}, \mathcal{O}(0)\right]=\left[\widetilde{S}_{\dot{\alpha} a}, \mathcal{O}(0)\right]=0$. In terms of the super-Poincaré generators this corresponds to

$$
Q_{\alpha \tilde{a}}^{\mathrm{L}}=i Q_{\alpha \tilde{a}}^{\mathrm{R}}, \quad Q_{\dot{\alpha}}^{\mathrm{L}} \tilde{a}=-i Q_{\dot{\alpha}}^{\mathrm{R}} \tilde{a}
$$

where $(\alpha, \dot{\alpha})$ are the explicit four-dimensional space-time spinor indices transverse to the trajectory in the $\mathrm{AdS}_{5}$ part and $(\tilde{a})$ are the $\mathrm{SO}(6)$ spinor indices for the remaining six dimensions.

The operators that satisfy (2.12) are described in detail in [29]. They are linear combinations of operators in the NS-NS and R-R sectors and have a fairly complicated form. However, their flat-space three-point functions were remarkably simple. In particular, the three-point function for two chiral primaries and a Konishi or Konishi-like operator (a primary operator at level one with a nonzero $R$-charge), is given by

$$
\left\langle V_{C, J_{1}} V_{C, J_{2}} V_{K, J_{3}}\right\rangle=g_{\mathrm{c}}^{3} \alpha^{\prime 2} \frac{\alpha_{1}^{2} \alpha_{2}^{2} \Sigma^{4} \tilde{\alpha}_{3}^{2} \tilde{\Sigma}^{2}}{\Delta_{1}^{2} \Delta_{2}^{2} \Delta_{3}^{4}},
$$

where

$$
\tilde{\Sigma}=\frac{1}{2}\left(\left|J_{1}\right|+\left|J_{2}\right|+\left|J_{3}\right|\right), \quad \tilde{\alpha}_{i}=\tilde{\Sigma}-\left|J_{i}\right| .
$$

In the special case where $J_{1}=-J_{2}=J, J_{3}=0$, this reduces to (1.6), where $\Delta=\Delta_{3}$.

\section{Extremal correlators and operator mixing}

In this section we compute the mixing between the double trace operator $\mathcal{O}_{J \bar{J}}$ and the Konishi operator $\mathcal{O}_{K}$. We first observe that the coupling in (1.4) near the extremal point $J=\Delta / 2 \approx \lambda^{1 / 4}$ is well approximated by

$$
\mathcal{G}_{123} \approx \frac{2^{3} \pi \sqrt{\lambda}}{N}
$$

where we have used the AdS/CFT dictionary for $g_{c}$ and $\alpha^{\prime}$. Using this, we also have that near the extremal point the three-point coupling coefficient in (1.2) is approximately

$$
\mathcal{C}_{123} \approx \frac{\lambda^{3 / 8}}{N} \frac{1}{2 J-\Delta} .
$$

The structure constants among physical primary operators must be finite, hence the pole in (3.2) signals that the operators renormalize and mix with each other. To find the mixing the double trace operator $\mathcal{O}_{J \bar{J}}(x)$ defined in (1.7) needs to be regulated. Here we use point-splitting and define the regulated operator

$$
\mathcal{O}_{J \bar{J}}^{\varepsilon}(x)=: \mathcal{O}_{J}(x+\varepsilon) \mathcal{O}_{\bar{J}}(x):=\mathcal{O}_{J}(x+\varepsilon) \mathcal{O}_{\bar{J}}(x)-\frac{1}{|\varepsilon|^{2 J}} .
$$


It is then clear that

$$
\left\langle\mathcal{O}_{J \bar{J}}^{\varepsilon}(x) \mathcal{O}_{J \bar{J}}^{\varepsilon}(y)\right\rangle=\frac{1}{|x-y|^{4 J}},
$$

while the mixing in the two-point function is

$$
\begin{aligned}
\left\langle\mathcal{O}_{J \bar{J}}^{\varepsilon}(x) \mathcal{O}_{K}(y)\right\rangle & =\left\langle\mathcal{O}_{J}(x+\varepsilon) \mathcal{O}_{\bar{J}}(x) \mathcal{O}_{K}(y)\right\rangle \\
& =\frac{\mathcal{C}_{123}}{|x-y|^{2 \Delta}|\varepsilon|^{2 J-\Delta}} \approx \frac{\lambda^{3 / 8}}{N} \frac{1}{(2 J-\Delta)|\varepsilon|^{2 J-\Delta}} \frac{1}{|x-y|^{2 \Delta}} .
\end{aligned}
$$

Note that the planar three-point function gives a nonplanar contribution to the two-point function of the Konishi operator with the double trace operator. As expected, in the limit of infinite $N$ there is no mixing between these operators.

$\mathcal{O}_{J \bar{J}}^{\varepsilon}(x)$ is just one of $M$ operators that have an equal two-point function with $\mathcal{O}_{K}(x)$, where $M$ is the dimension of the $J$-symmetric traceless representation of $\mathrm{SO}(6)$,

$$
M=\frac{(J+1)(J+2)^{2}(J+3)}{12} \approx \frac{J^{4}}{12} .
$$

Since $\mathcal{O}_{K}(x)$ is an $\mathrm{SO}(6)$ singlet, it only mixes with the singlet combination of the doubletrace operators,

$$
\mathcal{O}_{s, J}^{\varepsilon}(x)=\frac{1}{\sqrt{M}} \sum_{I=1}^{M}\left(\mathcal{O}_{I}(x+\varepsilon) \mathcal{O}_{I}(x)-\frac{1}{|\varepsilon|^{2 J}}\right),
$$

where $I$ refers to one of the states that make up the $J$-symmetric traceless representation. The two-point-function of the singlet double-trace and the Konishi operators is then

$$
\left\langle\mathcal{O}_{s, J}^{\varepsilon}(x) \mathcal{O}_{K}(y)\right\rangle \approx \frac{\lambda^{3 / 8} \sqrt{M}}{N} \frac{1}{(2 J-\Delta)|\varepsilon|^{2 J-\Delta}} \frac{1}{|x-y|^{2 \Delta}} .
$$

To cancel the divergence at the pole in (3.8) we take as the renormalized operators the combinations

$$
\begin{aligned}
\mathcal{O}_{K}^{\text {ren }} & =\mathcal{O}_{K}-\lim _{\Delta \rightarrow 2 J} \frac{\lambda^{3 / 8} \sqrt{M}}{N(2 J-\Delta)} \mathcal{O}_{s, J}^{\varepsilon}, \\
\mathcal{O}_{s, J}^{\varepsilon, \text { ren }} & =\mathcal{O}_{s, J}^{\varepsilon}+\frac{\lambda^{3 / 8} \sqrt{M} \log (\varepsilon \mu)}{N} \mathcal{O}_{K},
\end{aligned}
$$

where $\mu$ is some scale satisfying $\varepsilon \mu \ll 1$. Notice that to order $1 / N$ the pole in the threepoint function of $\mathcal{O}_{K}^{\text {ren }}$ with the two chiral primaries $\mathcal{O}_{J}$ and $\mathcal{O}_{\bar{J}}$ cancels. Expanding the pre-factors in (1.2), we find that the structure constant involving the renormalized operator at the pole is

$$
\mathcal{C}_{123}^{\mathrm{ren}} \approx-\frac{\lambda^{3 / 8} \log \lambda}{8 N}
$$

The pole in the three-point function of $\mathcal{O}_{s, J}^{\varepsilon, \text { ren }}$ with the same chiral primaries does not appear until order $1 / N^{2}$, and so to leading order in the $1 / N$ expansion it is also pole-free.

The point-splitting parameter $\varepsilon$ acts as a UV cutoff and leads to an anomalous dimension for the mixed operators. The anomalous dimension matrix at this order has the form

$$
\Gamma=\left(\begin{array}{cc}
0 & \delta_{s J, K} \\
\delta_{s J, K} & 0
\end{array}\right),
$$


where the off-diagonal elements are given by

$$
\delta_{s J, K}=\frac{1}{2} \varepsilon \frac{\partial}{\partial \varepsilon} \frac{\lambda^{3 / 8} \sqrt{M}}{N} \log (\varepsilon \mu)=\frac{\lambda^{3 / 8} \sqrt{M}}{2 N} .
$$

The eigenvalues of $\Gamma$ are then given by

$$
\delta_{ \pm}=\frac{\lambda^{3 / 8}}{2 N} \sqrt{M}
$$

Hence, at the cross-over point $J=\Delta / 2 \approx \lambda^{1 / 4}$, the mixed operators have dimensions

$$
\Delta_{ \pm}=2 J \pm \frac{\sqrt{M} \lambda^{3 / 8}}{2 N}
$$

and thus the splitting is $\Delta_{+}-\Delta_{-}$, giving the result in (1.8), which can be reexpressed as

$$
\Delta m \approx \frac{\sqrt{M} \lambda^{3 / 8}}{N} \approx \frac{\lambda^{7 / 8}}{2 \sqrt{3} N}
$$

Note that the structure constant in (3.10) is scheme dependent as we could have defined different renormalized operators by using other linear combinations of the bare operators. But this will not affect the structure constants between the chiral primaries and the final mixed operators that are the eigenstates of $\Gamma$.

We can compare the splitting to the leading correction to the double-trace dimension coming from supergravity, where it was found to be [37]

$$
\delta_{J J}=-\frac{2(J-1) J(J+2)}{N^{2}} .
$$

Hence the splitting, which scales as $J^{7 / 2} / N$, is much larger.

\section{Three-point couplings with spin}

\subsection{The operators and corresponding string states}

In this section we consider three-point functions where at least one of the operators is dual to a string state in the leading Regge trajectory. As in $[28,29]$, the correlators can have one or more chiral primaries,

$$
\mathcal{O}_{\mathrm{CP}}=C_{I_{1} I_{2} \ldots I_{J}} \operatorname{Tr}\left[\Phi^{I_{1}} \Phi^{I_{2}} \ldots \Phi^{I_{J}}\right]
$$

where $C_{I_{1} I_{2} \ldots I_{J}}$ is symmetric and traceless and $\Phi^{I}$ are the six scalars in $\mathcal{N}=4 \mathrm{SYM}$. These are states in the $[0, J, 0]$ representation of $\mathrm{SO}(6)$ and have the protected dimension $\Delta_{\mathrm{CP}}=J$. At strong coupling, these operators correspond to massless scalar vertex operators with nonzero Kaluza-Klein momentum, and have the following mixture of NS-NS and R-R modes $[28,29]$

$$
V_{C, k}=-\frac{1}{4}\left(W_{1, k}+\frac{1}{\sqrt{2}} W_{2, k}\right)
$$


The NS-NS and R-R vertices are given respectively by

$$
W_{1, k}=g_{\mathrm{c}} \varepsilon_{M \tilde{M}} \psi^{M} e^{-\phi} \tilde{\psi}^{\tilde{M}} e^{-\tilde{\phi}} e^{i k \cdot X}, \quad W_{2, k}=g_{\mathrm{c}} t_{A B} \tilde{\Theta}^{A} e^{-\frac{1}{2} \tilde{\phi}} \Theta^{B} e^{-\frac{1}{2} \phi} e^{i k \cdot X},
$$

with the following polarizations

$$
\varepsilon_{M \tilde{M}}=(-1)^{\sigma_{k}(\tilde{M})}\left(\eta_{M \tilde{M}}-\frac{k_{M} \bar{k}_{\tilde{M}}+k_{\tilde{M}} \bar{k}_{M}}{k \cdot \bar{k}}\right), t_{A B}=\left(\frac{\alpha^{\prime}}{2}\right)^{1 / 2}\left(C^{\dagger} i \Gamma^{0^{\prime}} \Gamma^{1^{\prime}} \Gamma^{2^{\prime}} \Gamma^{3^{\prime}} \not k\right)_{A B} .
$$

We define the untwisting factor as

$$
\sigma_{k}(M)= \begin{cases}1 & M=0^{\prime}, \ldots, 3^{\prime} \\ 0 & M=4^{\prime}, 5, \ldots, 9\end{cases}
$$

The primed indices denote a frame in which the directions $0^{\prime}, \ldots, 3^{\prime}$ are transverse to the momentum $k$.

The other operators considered in $[28,29]$ also transform in the $[0, J, 0]$ representation, but have bare dimension $\Delta_{0}=J+2$ [38]. Operators of this type include

$$
\mathcal{O}_{J}=\operatorname{Tr}\left[\Phi^{I} \Phi_{I} Z^{J}\right]+\ldots,
$$

where $Z=\left(\Phi_{5}+i \Phi_{6}\right) / \sqrt{2}$, and the ellipsis refers to different positions of the $\Phi_{I}$ in the trace, such that the corresponding magnon momenta lie at level one [38, 39]. For $J=0$ we have the Konishi operator

$$
\mathcal{O}_{\mathrm{K}}=\mathcal{O}_{J=0}=\operatorname{Tr}\left[\Phi^{I} \Phi_{I}\right],
$$

which at strong coupling has dimension $\Delta_{\mathrm{K}} \approx 2 \lambda^{1 / 4}$. These operators correspond at strong coupling to scalar vertex operators at the first massive string level, possibly with some Kaluza-Klein momentum. They can be written as

$$
V_{K, k}=-\frac{1}{16}\left(V_{1, k}+V_{2, k}+\frac{1}{\sqrt{2}} V_{3, k}\right),
$$

where $V_{1, k}$ and $V_{2, k}$ are the NS-NS vertices and $V_{3, k}$ is in the R-R sector

$$
\begin{aligned}
& V_{1, k}=g_{\mathrm{c}} \frac{2}{\alpha^{\prime}} \varepsilon_{M N ; \tilde{M} \tilde{N}} \psi^{M} i \partial X^{N} e^{-\phi} \tilde{\psi}^{\tilde{M}} i \bar{\partial} X^{\tilde{N}} e^{-\tilde{\phi}} e^{i k \cdot X} \\
& V_{2, k}=g_{\mathrm{c}} \alpha_{M N L ; \tilde{M} \tilde{N} \tilde{L}} \psi^{M} \psi^{N} \psi^{L} e^{-\phi} \tilde{\psi^{M}} \tilde{\psi} \tilde{N} \tilde{\psi}^{\tilde{L}} e^{-\tilde{\phi}} e^{i k \cdot X} \\
& V_{3, k}=\frac{2 g_{\mathrm{c}}}{\alpha^{\prime}}\left(i \bar{\partial} X^{M} \tilde{\Theta}-\frac{\alpha^{\prime}}{16} \tilde{\psi}^{M}(\not k \tilde{\psi} \tilde{\Theta})\right)^{A} e^{-\tilde{\phi} / 2} t_{M A ; N B}\left(i \partial X^{N} \Theta-\frac{\alpha^{\prime}}{16} \psi^{N}(\not k \psi \Theta)\right)^{B} e^{-\phi / 2} e^{i k X} .
\end{aligned}
$$

Defining the tensor $\hat{\eta}^{M N} \equiv \eta^{M N}-\frac{k^{M} k^{N}}{k^{2}}$, we can write the polarizations in the following way

$$
\begin{aligned}
\varepsilon_{M N ; \tilde{M} \tilde{N}} & =(-1)^{\sigma_{k}(\tilde{M})+\sigma_{k}(\tilde{N})}\left(\frac{1}{2}\left(\hat{\eta}_{M \tilde{M}} \hat{\eta}_{N \tilde{N}}+\hat{\eta}_{M \tilde{N}} \hat{\eta}_{N \tilde{M}}\right)-\frac{1}{9} \hat{\eta}_{M N} \hat{\eta}_{\tilde{M} \tilde{N}}\right), \\
\alpha_{M N L ; \tilde{M} \tilde{N} \tilde{L}} & =(-1)^{\sigma_{k}(\tilde{M})+\sigma_{k}(\tilde{N})+\sigma_{k}(\tilde{L})} \frac{1}{3 !^{2}}\left(\hat{\eta}_{M \tilde{M}} \hat{\eta}_{N \tilde{N}} \hat{\eta}_{L \tilde{L}} \mp(5 \text { permutations })\right), \\
t_{M A ; N B} & =(-1)^{\sigma_{k}(\tilde{M})}\left(\frac{\alpha^{\prime}}{2}\right)^{1 / 2}\left(C^{\dagger} i \Gamma^{0^{\prime}} \Gamma^{1^{\prime}} \Gamma^{2^{\prime}} \Gamma^{3^{\prime}} \not k\left(\hat{\eta}_{M N}-\frac{1}{9} \Gamma^{R} \Gamma^{S} \hat{\eta}_{M R} \hat{\eta}_{N S}\right)\right)_{A B} .
\end{aligned}
$$


In this work we will also consider relatives of the Lagrangian operator with $R$-charge $J$ which have protected dimension $\Delta_{\mathcal{L}_{J}}=4+J$

$$
\mathcal{O}_{\mathcal{L}_{J}}=\operatorname{Tr}\left[F_{\mu \nu} F^{\mu \nu} Z^{J}\right]+\ldots,
$$

where $F_{\mu \nu}$ is the field strength of $\mathcal{N}=4 \mathrm{SYM}$, and the ellipsis refers to different positions of the $F_{\mu \nu}$ in the trace, and also other scalar and spinor terms required by supersymmetry. At strong coupling these operators correspond to the ten-dimensional dilaton with some Kaluza-Klein momentum

$$
V_{\mathcal{L}, k}=\frac{g_{\mathrm{c}}}{\sqrt{8}}\left(\eta_{M \tilde{M}}-\frac{k_{M} \bar{k}_{\tilde{M}}+k_{\tilde{M}} \bar{k}_{M}}{k \cdot \bar{k}}\right) \psi^{M} e^{-\phi} \tilde{\psi}^{\tilde{M}} e^{-\tilde{\phi}} e^{i k \cdot X}
$$

Finally, an obvious extension to the results in [29] is to consider operators with spin. It is in general nontrivial to find the vertex operator for a generic operator with dimension $\Delta$ and spin $S$. However, if the operator is a special combination of the three following twist two operators [31]

$$
\operatorname{Tr}\left[F_{\mu \nu_{1}} D_{\nu_{2}} \ldots D_{\nu_{S-1}} F_{\nu_{S}}{ }^{\mu}\right], \operatorname{Tr}\left[\Phi_{I} D_{\nu_{1}} \ldots D_{\nu_{S}} \Phi^{I}\right], \operatorname{Tr}\left[\bar{\psi}_{\alpha} D_{\nu_{1}} \ldots D_{\nu_{S-1}} \Gamma_{\nu_{S}} \psi^{\alpha}\right]
$$

then the dual string state lies on the leading Regge trajectory at level $n=\frac{1}{2}(S-2)$ with scaling dimension $\Delta \approx 2 \sqrt{n} \lambda^{1 / 4}$.

As noted in [30], the vertex operator for a symmetric traceless state in the leading Regge trajectory is given by

$$
O_{n, k}=g_{\mathrm{c}}\left(\frac{2}{\alpha^{\prime}}\right)^{n} \varepsilon_{M_{1} \ldots M_{n+1} \tilde{M}_{1} \ldots \tilde{M}_{n+1}} \prod_{j=1}^{n}\left(i \partial X^{M_{j}}\right) \psi^{M_{n+1}} e^{-\phi} \prod_{j=1}^{n}\left(i \bar{\partial} X^{\tilde{M}_{j}}\right) \tilde{\psi}^{\tilde{M}_{n+1}} e^{-\tilde{\phi}} e^{i k \cdot X}
$$

with $\varepsilon_{M_{1} \ldots M_{S}}$ a totally symmetric and traceless tensor. To normalize the vertex operator we need only to compute its two-point function

$$
\left\langle O_{n, k} O_{n,-k}\right\rangle=\Gamma(S / 2)^{2} \varepsilon_{M_{1} \ldots M_{S}} \varepsilon^{M_{1} \ldots M_{S}} .
$$

The normalized vertex operator for a string state in the leading Regge trajectory is then

$$
V_{S, k}=\frac{g_{\mathrm{c}} 2^{S / 2-1}}{\Gamma\left(\frac{S}{2}\right) \alpha^{\prime S / 2-1}} \varepsilon_{M_{1} \ldots M_{S / 2}}^{\text {norm }} \tilde{M}_{1} \ldots \tilde{M}_{S / 2} \prod_{j=1}^{\frac{S-2}{2}}\left(i \partial X^{M_{j}}\right) \psi^{M_{S / 2}} e^{-\phi} \prod_{j=1}^{\frac{S-2}{2}}\left(i \bar{\partial} X^{\tilde{M}_{j}}\right) \tilde{\psi}^{\tilde{M}_{S / 2}} e^{-\tilde{\phi}} e^{i k \cdot X} .
$$

\subsection{The building blocks}

Three-point functions of operators with arbitrary spin can be written as a linear combination of a finite number of conformally invariant building blocks [40-42], which have been rederived in [43] using the embedding formalism

$$
C\left(\left\{x_{i}\right\}\right)=\frac{Q\left(\left\{x_{i}\right\}\right)}{\left(x_{12}^{2}\right)^{\frac{\tau_{1}+\tau_{2}-\tau_{3}}{2}}\left(x_{13}^{2}\right)^{\frac{\tau_{1}+\tau_{3}-\tau_{2}}{2}}\left(x_{23}^{2}\right)^{\frac{\tau_{2}+\tau_{3}-\tau_{1}}{2}}},
$$


where $\tau_{i}=\Delta_{i}+S_{i}$ and $Q\left(\left\{x_{i}\right\}\right)$ is a polynomial built out of six building blocks

$$
V_{1} \equiv V_{1,23}, \quad V_{2} \equiv V_{2,31}, \quad V_{3} \equiv V_{3,12}, \quad H_{12}, \quad H_{13}, \quad H_{23},
$$

which encode the space-time dependence

$$
V_{i, j k}=\frac{1}{x_{j k}^{2}}\left(x_{i j}^{2}\left(z_{i} \cdot x_{i k}\right)-x_{i k}^{2}\left(z_{i} \cdot x_{i j}\right)\right), \quad H_{i j}=x_{i j}^{2}\left(z_{i} \cdot z_{j}\right)-2\left(z_{i} \cdot x_{i j}\right)\left(z_{j} \cdot x_{i j}\right) .
$$

Here we encode symmetric tensors by polynomials obtained by contracting the tensor with a reference vector $z_{i}^{\mu}$. We can recover the tensor by applying the symmetric traceless projector

$$
P_{\mu_{1} \ldots \mu_{S}}^{i}=\frac{1}{(S !)^{2}} D_{\mu_{1}}^{i} \ldots D_{\mu_{S}}^{i}, \quad D_{\mu}^{i}=\left(1+z_{i} \cdot \frac{\partial}{\partial z_{i}}\right) \frac{\partial}{\partial z_{i}^{\mu}}-\frac{1}{2} z_{i \mu} \frac{\partial^{2}}{\partial z_{i} \cdot \partial z_{i}} .
$$

The polynomial $Q\left(\left\{x_{i}\right\}\right)$ is then written as the following sum

$$
P_{\mu_{1} \ldots \mu_{S_{1}}}^{1} P_{\nu_{1} \ldots \nu_{S_{2}}}^{2} P_{\rho_{1} \ldots \rho_{S_{3}}}^{3} \sum_{i, j, k \in \mathcal{I}} C_{i j k} V_{1}^{S_{1}-i-j} V_{2}^{S_{2}-i-k} V_{3}^{S_{3}-j-k} H_{12}^{i} H_{13}^{j} H_{23}^{k},
$$

where $C_{i j k}$ are the structure constants and the summation range is

$$
\mathcal{I}=\left\{i, j, k \in \mathbb{N}_{0}: S_{1}-i-j \geq 0, \quad S_{2}-i-k \geq 0, \quad S_{3}-j-k \geq 0\right\} .
$$

We can reproduce these structures with our holographic procedure. In [30] Schlotterer derived the coupling of three Regge open superstring states at levels $n_{1}, n_{2}$ and $n_{3} .{ }^{1}$ We can apply this result directly to the left and right movers separately. The three-point function for the left movers is,

$$
\begin{aligned}
\left\langle O_{n_{1}} O_{n_{2}} O_{n_{3}}\right\rangle_{L}= & {\sqrt{\alpha^{\prime} / 2}}^{s_{1}+s_{2}+s_{3}} n_{1} ! n_{2} ! n_{3} ! \sum_{i, j, k \in \mathcal{I}} \frac{\left(\alpha^{\prime} / 2\right)^{-i-j-k}\left(i s_{3}+j s_{2}+k s_{1}-i j-i k-j k\right)}{i ! j ! k !\left(s_{1}-i-j\right) !\left(s_{2}-i-k\right) !\left(s_{3}-j-k\right) !} \\
& \times\left(\epsilon^{1} \cdot k_{2}^{s_{1}-i-j}\right)\left(\epsilon^{2} \cdot k_{3}^{s_{2}-i-k}\right)\left(\epsilon^{3} \cdot k_{1}^{s_{3}-j-k}\right) \eta_{12}^{i} \eta_{13}^{j} \eta_{23}^{k},
\end{aligned}
$$

where $s_{i}=n_{i}+1$. We used the following shorthand notations

$$
\begin{aligned}
\epsilon^{i} \cdot k_{j}^{p} & =\epsilon_{M_{1} \ldots M_{p} M_{p+1} \ldots M_{s_{i}}}^{i} k_{j}^{M_{1}} \ldots k_{j}^{M_{p}}, \\
\epsilon^{i} \epsilon^{j} \eta_{i j}^{k} & =\epsilon_{M_{1} \ldots M_{s_{i}}}^{i} \epsilon_{N_{1} \ldots N_{s_{j}}}^{j} \eta^{M_{1} N_{1}} \ldots \eta^{M_{k} N_{k}},
\end{aligned}
$$

and the summation range is the same as in (4.22). We notice then that the superstring amplitude of three closed spin states with polarizations $\varepsilon_{1}, \varepsilon_{2}$ and $\varepsilon_{3}$ will be a sum of terms of the form

$$
\left(\epsilon^{1} \cdot k_{2}^{S_{1}-i-j}\right)\left(\epsilon^{2} \cdot k_{3}^{S_{2}-i-k}\right)\left(\epsilon^{3} \cdot k_{1}^{S_{3}-j-k}\right) \eta_{12}^{i} \eta_{13}^{j} \eta_{23}^{k},
$$

which we represent diagrammatically in figure 1 . These superstring structures from the coupling of the three string states at the intersection point in the bulk are in fact related to the boundary CFT building blocks.

\footnotetext{
${ }^{1}$ This work is an extension of [44] which addresses a related problem in the context of open bosonic strings.
} 


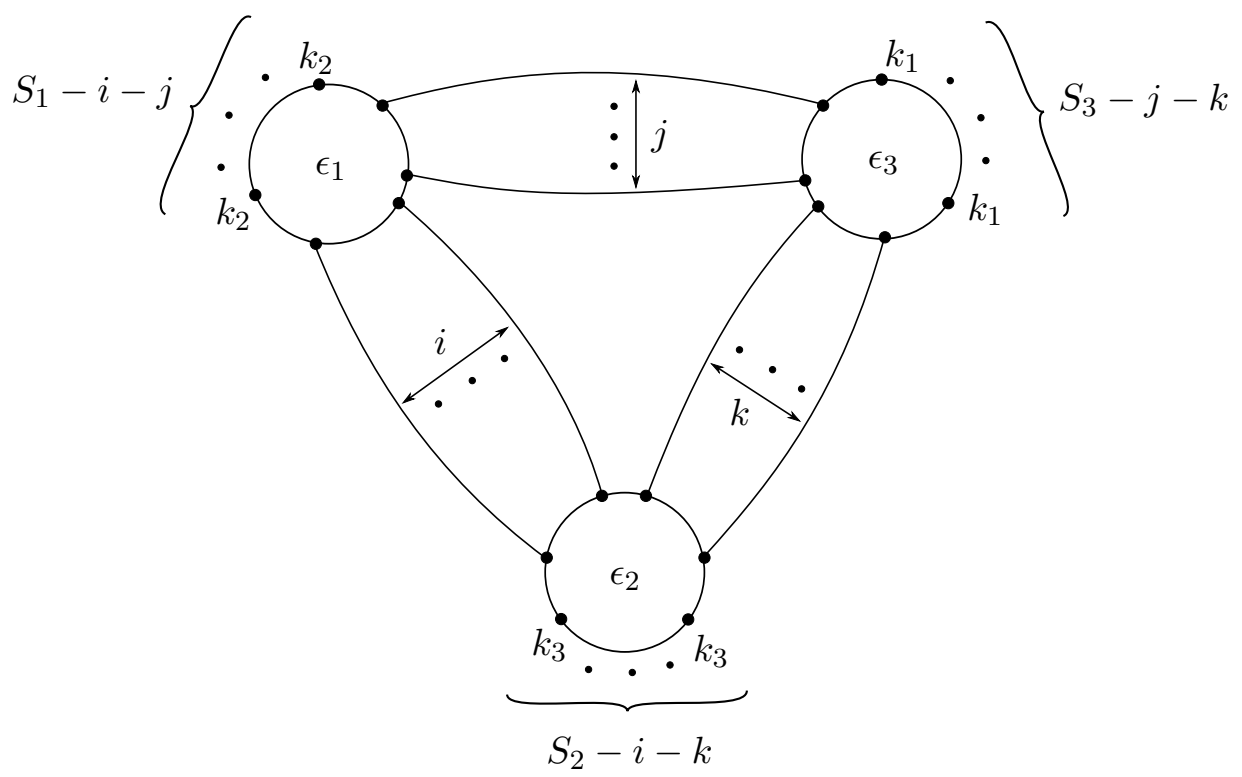

Figure 1. We represent each polarization $\epsilon_{i}$ by a circle with $S_{i}$ indices. The lines connecting two polarization tensors correspond to contractions of their indices. The leftover indices are contracted with the momenta. The allowed structures correspond to the different ways of connecting the three polarization tensors.

To understand this relation between the two sets of building blocks, it will be enough to restrict ourselves to the $\mathrm{AdS}_{3}$ space defined by the bulk direction and the plane on the boundary defined by the three endpoints of the strings. If the polarization is orthogonal to any of those directions then $\varepsilon^{i} \cdot k_{j}$ vanishes trivially and $\varepsilon^{i} \cdot \varepsilon^{j}$ remains proportional to $H_{i j}$. On the other hand, if the polarizations live in some direction inside the $\mathrm{AdS}_{3}$ defined above then we must be more careful. At the intersection point the polarization of each string is orthogonal to the momentum which, following [28], is given by

$$
k^{\mu}=\frac{1}{\sqrt{2}}\left(\kappa\left\langle P^{\mu}\right\rangle-\kappa^{-1}\left\langle K^{\mu}\right\rangle\right), \quad k^{z}=-\langle D\rangle,
$$

with $\left\langle P^{\mu}\right\rangle,\langle D\rangle,\left\langle K^{\mu}\right\rangle$ and $\kappa$ defined in (2.1), (2.4), and (2.6) when one takes the intersection point to the origin. In this case, the momentum at the intersection point can be written in the following way

$$
k_{i}^{\mu}=-2 \frac{\sqrt{\alpha_{1} \alpha_{2} \alpha_{3} \Sigma}\left|x_{12}\right|\left|x_{23}\right|\left|x_{13}\right|}{F}\left(\alpha_{k} \frac{x_{i j}^{\mu}}{x_{i j}^{2}}+\alpha_{j} \frac{x_{i k}^{\mu}}{x_{i k}^{2}}\right), \quad k_{i}^{z}=\Delta_{i}-2 \frac{\alpha_{j} \alpha_{k} \Sigma x_{j k}^{2}}{F} .
$$

Since the polarization is orthogonal to the momentum at the intersection point, it is useful to change to a basis $\left\{\vec{O}_{i}, \vec{P}_{i}\right\}$ in the plane transverse to the momentum. If we let $\vec{k}_{i}^{B}$ be the projection of $\vec{k}_{i}$ to the boundary then we can write the basis vectors as

$$
\vec{O}_{i}=\vec{k}_{i} \times \vec{k}_{i}^{B}, \quad \vec{P}_{i}=\vec{k}_{i} \times \vec{O}_{i} .
$$

We denote this change of basis by $M_{i}^{I}$. When one looks at the propagation of the string from the intersection point to the boundary, one sees that $\vec{O}_{i}$ stays the same, but $\vec{P}_{i}$ becomes 
proportional to $\vec{k}_{i}^{B}$. Finally, we return from the basis $\left\{\vec{O}_{i}, \vec{k}_{i}^{B}\right\}$ to the canonical one at the boundary through another change of basis $M_{i}^{B}$. The relation between the polarization at the intersecton point and at the boundary is then given by

$$
\left(\varepsilon_{i}^{I}\right)^{M}=\left(M_{i}^{I} M_{i}^{B}\right)^{M}\left(\varepsilon_{i}^{B}\right)^{\mu},
$$

where we denoted the polarization at the intersection point by $\varepsilon_{i}^{I}$ and the one at the boundary by $\varepsilon_{i}^{B}$. The polarization $\varepsilon_{i}^{I}$ is symmetric and traceless, which leads to a polarization at the boundary with the same properties. $\varepsilon_{i}^{B}$ will then act as a symmetric and traceless projector ensuring the right tensor structure of $Q\left(\left\{x_{i}\right\}\right)$. The structure at the intersection point $\varepsilon_{i}^{I} \cdot k_{j}$ then corresponds to one of the CFT building blocks at the boundary

$$
\varepsilon_{i}^{I} \cdot k_{j}=\left(\varepsilon_{i}^{B}\right)^{\mu}\left(-2 \frac{\sqrt{\alpha_{1} \alpha_{2} \alpha_{3} \Sigma} x_{j k}^{2}}{\left|x_{12}\right|\left|x_{13}\right|\left|x_{23}\right| \Delta_{i}} P_{\mu}^{i} V_{i}\right) .
$$

Analogously, the structure $\varepsilon_{i}^{I} \cdot \varepsilon_{j}^{I}$ becomes a combination of the CFT building blocks at the boundary

$$
\varepsilon_{i}^{I} \varepsilon_{j}^{I} \eta_{i j}=\left(\varepsilon_{i}^{B}\right)^{\mu} \frac{1}{x_{i j}^{2}} P_{\mu}^{i} P_{\nu}^{j}\left(H_{i j}+2 \frac{\alpha_{i} \alpha_{j}}{\Delta_{i} \Delta_{j}} V_{i} V_{j}\right)\left(\varepsilon_{j}^{B}\right)^{\nu} .
$$

Putting all the elements together, terms of the form (4.25) now become proportional to

$$
\prod_{\substack{i<j \\ k \neq i, j}}\left(x_{i j}^{2}\right)^{-\frac{S_{i}+S_{j}-S_{k}}{2}} \prod_{i} V_{i}^{S_{i}-\sum_{j \neq i} n_{i j}} \prod_{i<j}\left(H_{i j}+2 \frac{\alpha_{i} \alpha_{j}}{\Delta_{i} \Delta_{j}} V_{i} V_{j}\right)^{n_{i j}}
$$

Notice that the spacetime dependence from the string theory amplitude combines with the one obtained from the propagation of the strings in AdS, $\left(x_{12}^{2}\right)^{-\alpha_{3}}\left(x_{13}^{2}\right)^{-\alpha_{2}}\left(x_{23}^{2}\right)^{-\alpha_{1}}$, to produce the spacetime behaviour expected from conformal symmetry in (4.17).

It will also be useful to work out the case where one operator is a scalar, for which we set $S_{1}=0$. With no loss of generality we choose $S_{2} \leq S_{3}$, so the amplitude will reduce to a sum over $\left(S_{2}+1\right)$ structures with coefficients $\mathcal{F}_{k}$

$$
\sum_{k=0}^{S_{2}}\left(\alpha^{\prime} / 2\right)^{\frac{S_{2}}{2}+\frac{S_{3}}{2}-k}\left(\epsilon^{2} \cdot k_{3}^{S_{2}-k}\right)\left(\epsilon^{3} \cdot k_{1}^{S_{3}-k}\right) \eta_{23}^{k} \mathcal{F}_{k} .
$$

Following our analysis on the equivalence of the two sets of building blocks, the three-point function of a scalar with two spin states becomes

$\mathcal{D} \sum_{k=0}^{S_{2}} \frac{\left(2 \alpha^{\prime} \alpha_{1} \alpha_{2} \alpha_{3} \Sigma\right)^{\frac{S_{2}}{2}+\frac{S_{3}}{2}-k}}{\Delta_{2}^{S_{2}-k} \Delta_{3}^{S_{3}-k}} V_{2}^{S_{2}-k} V_{3}^{S_{3}-k}\left(H_{23}+\frac{2 \alpha_{2} \alpha_{3}}{\Delta_{2} \Delta_{3}} V_{2} V_{3}\right)^{k} \mathcal{F}_{k}=\sum_{k=0}^{S_{2}} C_{k} V_{2}^{S_{2}-k} V_{3}^{S_{3}-k} H_{23}^{k}$,

where $\mathcal{D}$ denotes both the contribution from the propagation of the strings in AdS (1.2) and the coupling (1.4)

$$
\mathcal{D}=\frac{\sqrt{\left(\Delta_{1}-1\right)\left(\Delta_{2}-1\right)\left(\Delta_{3}-1\right)}}{2^{5 / 2} \pi} \frac{\Gamma\left(\alpha_{1}\right) \Gamma\left(\alpha_{2}\right) \Gamma\left(\alpha_{3}\right) \Gamma(\Sigma-2)}{\Gamma\left(\Delta_{1}\right) \Gamma\left(\Delta_{2}\right) \Gamma\left(\Delta_{3}\right)} \frac{8 \pi}{g_{\mathrm{c}}^{2} \alpha^{\prime}}\left\langle\psi_{J_{1}} \psi_{J_{2}} \psi_{J_{3}}\right\rangle,
$$


and the structure constants are

$$
C_{k}=\mathcal{D} \frac{\left(2 \alpha^{\prime} \alpha_{1} \alpha_{2} \alpha_{3} \Sigma\right)^{S_{2} / 2+S_{3} / 2-k}}{\Delta_{2}^{S_{2}-k} \Delta_{3}^{S_{3}-k}} \sum_{l=k}^{S_{2}}\left(\begin{array}{l}
l \\
k
\end{array}\right)\left(\alpha^{\prime} \alpha_{1} \Sigma\right)^{k-l} \mathcal{F}_{l} .
$$

In the other case of interest, we let two of the operators be scalars, hence $S_{1}=S_{2}=0$. There is now only the structure $\left(\alpha^{\prime} / 2\right)^{S_{3} / 2}\left(\epsilon^{3} \cdot k_{1}^{S_{3}}\right) \mathcal{F}$, with the coefficient $\mathcal{F}$ coming from the superstring amplitude. The structure constant is then given by

$$
C=\mathcal{D} \frac{\left(2 \alpha^{\prime} \alpha_{1} \alpha_{2} \alpha_{3} \Sigma\right)^{S_{3} / 2}}{\left(\Delta_{3}\right)^{S_{3}}} \mathcal{F}
$$

\subsection{Structure constants}

Now that we have the necessary ingredients, we can compute the three-point coupling for different string states.

Two massless scalars and one Regge spin. We start by computing the three-point functions having two massless scalars, which we can compare with previous results in the literature. When the two massless scalars are dilatons, we can insert the polarizations defined in (4.12) into the results of appendix A, thus giving the string amplitude between two dilatons and one Regge spin

$$
\left\langle V_{\mathcal{L}} V_{\mathcal{L}} V_{S}\right\rangle=\frac{\left(2 \alpha^{\prime} \alpha_{1} \alpha_{2} \alpha_{3} \Sigma\right)^{S / 2}}{\left(\Delta_{3}\right)^{S} \Gamma(S / 2)} V_{3}^{S}
$$

Analogously, if we consider the massless scalars to be two chiral primaries, then we use the polarizations defined in (4.4), obtaining the following amplitude for two chiral operators with $R$-charges $J_{1}$ and $J_{2}$ and one Regge spin with some $R$-charge $J_{3}$

$$
\left\langle V_{C} V_{C} V_{S}\right\rangle=\frac{\tilde{\alpha}_{3}^{2} \tilde{\Sigma}^{2}\left(2 \alpha^{\prime} \alpha_{1} \alpha_{2} \alpha_{3} \Sigma\right)^{S / 2}}{J_{1}^{2} J_{2}^{2}\left(\Delta_{3}\right)^{S} \Gamma(S / 2)} V_{3}^{S}
$$

When the two chirals have opposite R-charge and the Regge spin has no $R$-charge, the expression (4.39) becomes (4.38). In this case we can put this result into (1.2) and (1.4), and taking the asymptotics for large 't Hooft coupling, we find the structure constant for two massless scalars with dimension $\Delta$ and a Regge state with spin $S$ and dimension $\Delta_{S} \approx \sqrt{2(S-2)} \lambda^{1 / 4}$

$$
C_{\mathcal{L L} S}=\frac{(-1)^{\Delta} \pi^{3 / 2}(S-2)^{(S-3) / 2+\Delta} \lambda^{-1 / 4+\Delta / 2}}{2^{S+\Delta-7 / 2} \Gamma(S / 2) \Gamma(\Delta-1) \Gamma(\Delta) N} 2^{-\Delta_{S}} \csc \left(\pi \Delta_{S} / 2\right) .
$$

This reproduces the results obtained in [31], where the authors used Mellin transforms. Note also the presence of poles in the factor $\csc \left(\pi \Delta_{S} / 2\right)$ which are related to mixing with double-trace operators as discussed in the previous section.

Another case we consider is when both scalars have the same dimension $\Delta$, but with the Regge spin also at the massless level, which corresponds to a relative of the graviton 
with some $R$-charge $J_{g}$. When $1 \ll\left|J_{g}\right| \ll \Delta$ then (4.39) becomes simply (4.38) and the structure constant we obtain in that limit is

$$
C_{C C G}=\frac{\sqrt{\pi}}{2^{3+J} N} \Delta,
$$

again obtaining agreement with [31] for large scaling dimensions.

If we wish to compute the coupling between three states with $R$-charges of the same magnitude, then we can compare with [45] where the authors used supergravity to compute the coupling between two chirals and a graviton with some Kaluza-Klein momentum. For chirals with dimension $J$ and a graviton with $R$-charge $J_{g}=J$ we get

$$
C_{C C G}=\frac{J^{3 / 2}}{8 N}\left\langle C^{J} C^{J} C^{J}\right\rangle
$$

where $\left\langle C^{J_{1}} C^{J_{2}} C^{J_{3}}\right\rangle$ is the unique $\mathrm{SO}(6)$ invariant that can be formed from the $C^{J_{i}}$ appearing in the definition of the spherical harmonics on $S^{5}$

$$
\psi_{J}=\frac{2^{\frac{J-1}{2}} \sqrt{(J+1)(J+2)}}{\pi^{3 / 2}} C_{i_{1} \ldots i_{J}}^{J} x^{i_{1}} \ldots x^{i_{J}} .
$$

The structure constant in (4.42) agrees with [45] in the limit of large scaling dimensions. For completeness we have also computed the coupling between one dilaton operator, a chiral and an operator in the leading Regge trajectory and have obtained a vanishing structure constant at leading order.

One massive scalar, one massless scalar and one Regge spin. If we consider the three-point function of a Konishi operator, a dilaton with some $R$-charge $J$ and a Regge spin with the opposite $R$-charge, we can use the results of appendix A and plug in the polarizations from (4.10), (4.12), obtaining the amplitude

$$
\left\langle V_{K} V_{\mathcal{L}} V_{S}\right\rangle=-\frac{(S-2)}{2^{5} \sqrt{2}} \frac{\left(2 \alpha^{\prime} \alpha_{1} \alpha_{2} \alpha_{3} \Sigma\right)^{S / 2}}{\left(\Delta_{3}\right)^{S} \Gamma(S / 2)} V_{3}^{S} .
$$

Notice that this amplitude vanishes when we take the Regge spin to $S=2$. Going to the limit of large 't Hooft coupling, the structure constant in the case of a Regge spin at the first mass level is

$$
C_{K \mathcal{L} S}=-\frac{J^{4} \sqrt{\pi}}{2^{9+J} \sqrt{2} N} \lambda^{-3 / 4}
$$

If instead of the dilaton we consider a chiral primary, then we use the polarizations of (4.4), (4.10) in the results of appendix A. When the spin operator has $S=2$, the structure constant also vanishes at leading order. When considering a Konishi, one chiral with $R$-charge $J$ and a massive Regge spin the amplitude for $J \ll \lambda^{1 / 4}$ is

$$
\left\langle V_{K} V_{C} V_{S}\right\rangle=\frac{(S-2)}{2^{6}} \frac{\left(2 \alpha^{\prime} \alpha_{1} \alpha_{2} \alpha_{3} \Sigma\right)^{S / 2}}{\left(\Delta_{3}\right)^{S} \Gamma(S / 2)} V_{3}^{S} .
$$

When the Regge spin is at the first massive level we obtain

$$
C_{K C S}=\frac{J^{4} \sqrt{\pi}}{2^{6+J} N} \lambda^{-3 / 4} .
$$


Two massive scalars and one Regge spin. The two massive scalars we consider here are the Konishi-like operators with the vertex operators (4.8). To compute the correlation function between two of this operators and one Regge spin we use the results derived in appendix A and plug in the polarization (4.10) thus obtaining, in the case of a massive spin, where all operators can be taken to have no $R$-charge

$$
\left\langle V_{K} V_{K} V_{S}\right\rangle=\frac{(S+14)^{2}}{2^{8}} \frac{\left(2 \alpha^{\prime} \alpha_{1} \alpha_{2} \alpha_{3} \Sigma\right)^{S / 2}}{\left(\Delta_{3}\right)^{S} \Gamma(S / 2)} V_{3}^{S}
$$

In the limit of large 't Hooft coupling, we get for the structure constant of two Konishi operators with dimension $\Delta_{K} \approx 2 \lambda^{1 / 4}$ and a massive Regge spin of dimension $\Delta_{S}$ satisfying the triangle inequalities

$$
C_{K K S}^{t}=\frac{(10-S)^{\frac{S-1}{2}}(S+14)^{2} \sqrt{\pi} \lambda^{\frac{1}{4}}}{2^{\frac{7}{2}+S} N \Gamma(S / 2)} 2^{-2 \Delta_{K}-\Delta_{S}}(2-\sqrt{n})^{\Delta_{K}-\Delta_{S} / 2}(2+\sqrt{n})^{-2+\Delta_{K}+\Delta_{S} / 2}
$$

where $n$ is the mass level of the Regge spin. If the massive Regge spin does not obey the triangle inequalities then the structure constant acquires poles,

$$
C_{K K S}^{n t}=C_{K K S}^{t} \times \frac{\csc \left((\sqrt{n}-2) \pi \lambda^{1 / 4}\right)}{2} .
$$

When the Regge spin is at the massless level and has an $R$-charge $J \ll \lambda^{1 / 4}$, we get

$$
\left\langle V_{K} V_{K} V_{G}\right\rangle=\frac{\left(2 \alpha^{\prime} \alpha_{1} \alpha_{2} \alpha_{3} \Sigma\right)^{S / 2}}{\left(\Delta_{3}\right)^{S} \Gamma(S / 2)} V_{3}^{S}
$$

Then the structure constant of a Konishi operator, a Konishi-like operator with some $R$-charge $J$ and a relative of the graviton with the opposite $R$-charge is

$$
C_{K K G}=\frac{\sqrt{\pi}}{2^{2+J} N} \lambda^{1 / 4},
$$

in the limit of large dimensions.

One massless scalar and two Regge spins. When there are two spins with $S_{2} \leq S_{3}$, there are $\left(S_{2}+1\right)$ different structure constants in (4.33). In appendix A we have computed the amplitude for a dilaton with two spins (A.18), which we can rewrite as

$$
\left\langle V_{\mathcal{L}} V_{S_{2}} V_{S_{3}}\right\rangle=\sum_{k=0}^{S_{2}}\left(\alpha^{\prime} / 2\right)^{S_{2} / 2+S_{3} / 2-k}\left(\epsilon^{2} \cdot k_{3}^{S_{2}-k}\right)\left(\epsilon^{3} \cdot k_{1}^{S_{3}-k}\right) \eta_{23}^{k} \mathcal{D}_{k}
$$

with the coefficients given by

$$
\mathcal{D}_{k}=\frac{1}{\sqrt{8}} \frac{1}{\prod_{i=2}^{3}\left(\frac{S_{i}-2}{2}\right) !\left(\frac{S_{i}}{2}\right)_{-\lfloor Y\rfloor-1}} \sum_{j=0}^{\lfloor Y\rfloor} \frac{2^{2-2 Y}(2 j+2) !}{j !(j+1) !(2 Y-2 j) !} \prod_{i=2}^{3} \frac{\left(\frac{S_{i}}{2}-j-2\right)_{j+1-\lfloor Y\rfloor}}{\left(S_{i}-2 j-4\right)_{2 j-2 Y+1}}
$$


where $Y=k / 2-2,\lfloor Y\rfloor$ denotes the largest integer not greater than $Y$ and $(a)_{m}$ is the Pochhammer symbol, $(a)_{m}=\Gamma(a+m) / \Gamma(a)$. Analogously, we have computed the amplitude for a chiral operator of dimension $\Delta$ with two spins (A.19), which we can rewrite as

$$
\left\langle V_{C} V_{S_{2}} V_{S_{3}}\right\rangle=\sum_{k=0}^{S_{2}}\left(\alpha^{\prime} / 2\right)^{S_{2} / 2+S_{3} / 2-k}\left(\epsilon^{2} \cdot k_{3}^{S_{2}-k}\right)\left(\epsilon^{3} \cdot k_{1}^{S_{3}-k}\right) \eta_{23}^{k} \mathcal{C}_{k}
$$

where

$$
\mathcal{C}_{k}=-\frac{\mathcal{D}_{k}}{\sqrt{2}}+\sum_{l=0}^{k} \frac{n_{2} ! n_{3} ! \delta}{4(l-1) !(k-l-1) !\left(s_{2}-l\right) !\left(s_{3}-l\right) !\left(s_{2}-k+l\right) !\left(s_{3}-k+l\right) !},
$$

and the values of $\delta$ for two massive spins, one massless spin of dimension $\Delta$ and one massive spin, and two spins with $S=2$ are

$$
\delta_{S S}=\frac{\Delta^{2}}{4 \sqrt{\lambda}}, \quad \delta_{G S}=0, \quad \delta_{G G}=-\frac{4 \alpha_{1} \alpha_{2} \alpha_{3} \Sigma}{\Delta^{2} \sqrt{\lambda}},
$$

respectively. Taking the expression for the structure constant (4.36) for a chiral primary with scaling dimension $\Delta$ and two massive Regge states with the same spin $S$, in the limit of large coupling constant we have

$$
C_{\mathcal{L} S S}^{k}=\frac{2^{k-S} \Delta^{2 S-2 k} \lambda^{k / 2-S / 2+1 / 4} \Gamma(\Delta / 2)^{2}}{N \sqrt{(\Delta-1)(S-2)} \Gamma(\Delta-1)} \sum_{l=k}^{S}\left(\begin{array}{l}
l \\
k
\end{array}\right)(2 S-4)^{k-l} \mathcal{C}_{l} .
$$

If we take one of the Regge spins to have $S=2$ and the opposite $R$-charge of the scalar, then for $\Delta \ll \lambda^{1 / 4}$ the structure constant is

$$
C_{\mathcal{L} G S}^{k}=\frac{(-1)^{\Delta} \pi^{\frac{3}{2}}(S-2)^{\frac{S+2 \Delta-3 k+5}{2}} \Delta^{k-3} \lambda^{\frac{2 \Delta-k+5}{4}} \csc \left(\pi \Delta_{S} / 2\right)}{2^{S+\Delta-\frac{3 k+1}{2}+\Delta_{S}} N \Gamma(\Delta-1) \Gamma(\Delta+3)} \sum_{l=k}^{2}\left(\begin{array}{l}
l \\
k
\end{array}\right)\left(\frac{S-2}{2}\right)^{k-l} \mathcal{C}_{l} .
$$

Finally, if both operators in the Regge trajectory are at the massless level and have dimension $\Delta$ as the massless scalar then

$$
C_{\mathcal{L} G G}^{k}=\frac{8^{k-2} \Delta^{7 / 2-2 k} \lambda^{\frac{k-1}{2}}}{3^{k-1} N}\left\langle C^{\Delta} C^{\Delta} C^{\Delta}\right\rangle\left(\begin{array}{l}
2 \\
k
\end{array}\right)\left(\frac{4 \sqrt{\lambda}}{3 \Delta^{2}}\right)^{S-k} \mathcal{C}_{S}
$$

One massive scalar and two Regge spins. In appendix A we have computed the two correlators that make up the amplitude for a Konishi with two spins (A.22), (A.25), which we can put together as

$$
\left\langle V_{K} V_{S_{2}} V_{S_{3}}\right\rangle=\sum_{k=0}^{S_{2}}\left(\alpha^{\prime} / 2\right)^{S_{2} / 2+S_{3} / 2-k}\left(\epsilon^{2} \cdot k_{3}^{S_{2}-k}\right)\left(\epsilon^{3} \cdot k_{1}^{S_{3}-k}\right) \eta_{23}^{k} \mathcal{K}_{k}
$$

The coefficient $\mathcal{K}_{k}$ is quite lengthy so we refer the reader to (A.28). Taking the expression for the structure constant (4.36) for a Konishi and two massive Regge states with the same spin $S$, in the limit of large coupling constant

$C_{K S S}^{k}=\frac{\sqrt{\pi}(2 \sqrt{n}-1)^{-\frac{1}{2}+\Delta_{S}-\Delta_{K} / 2}(2 \sqrt{n}+1)^{-\frac{5}{2}+\Delta_{S}+\Delta_{K} / 2}}{2^{\Delta_{S}+\Delta_{K}-3}(S-2)^{S-k-1+\Delta_{S}} N} \lambda^{1 / 4} \sum_{l=k}^{S}\left(\begin{array}{l}l \\ k\end{array}\right)(2 S-5)^{k-l} \mathcal{K}_{l}$, 
where $n$ is the mass level of the Regge spins. If we take one of the Regge spins to be at the massless level with scaling dimension $\Delta \ll \lambda^{1 / 4}$ and the other at the first massive level then the structure constant becomes

$$
C_{K G S}^{k}=\frac{\Delta^{\frac{9}{2}-k} \lambda^{\frac{k-3}{4}} \Gamma(\Delta / 2)^{2}}{2 \sqrt{2} N \Gamma(\Delta)} \sum_{l=k}^{2}\left(\begin{array}{l}
l \\
k
\end{array}\right)\left(\frac{\lambda^{1 / 4}}{\Delta}\right)^{l-k} \mathcal{K}_{l} .
$$

Finally, the structure constant for one Konishi and two states with spin $S=2$ and dimension $\Delta \ll \lambda^{1 / 4}$ is

$$
C_{K G G}^{k}=\frac{(-1)^{\Delta} \pi^{\frac{3}{2}} \Delta^{1+2 k} \lambda^{\frac{1+2 \Delta-2 k}{4}}}{2^{k-3+\Delta_{K}} N \Gamma(\Delta+1)^{2}} \csc \left(\pi \Delta_{K} / 2\right) \sum_{l=k}^{2}\left(\begin{array}{l}
l \\
k
\end{array}\right)(-1)^{l-k} \mathcal{K}_{l} .
$$

\section{Discussion}

In the first part of this article we studied how the poles appearing in extremal three-point functions relate to the mixing of single- and double-trace operators of $\mathcal{N}=4 \mathrm{SYM}$. We have made this relation quantitative for the case of the Konishi operator and the double-trace operator made of chiral primaries with large $R$-charge.

In the paper's second part we computed three-point functions involving higher spin states. For correlators with symmetric traceless operators, conformal symmetry allows for a number of different spacetime dependent structures. Here we have successfully matched the building blocks appearing in the superstring amplitudes with the structures allowed by conformal symmetry. The set of operators we considered are the chiral primaries, the scalar primaries dual to string states at the first massive level, Lagrangian-like operators dual to the dilaton with some Kaluza-Klein momentum and twist two operators dual to strings in the leading Regge trajectory. We have also successfully matched our results for the three-point functions of two massless scalars and a spin state in the leading Regge trajectory with recent calculations using the Mellin amplitude formalism, and older supergravity calculations. Having checked with these known cases we went on to compute new correlation functions involving two higher spin states and Konishi operators.

For the future, there are many interesting directions to explore. For example, it would be interesting to generalize our procedure to include operators with small scaling dimensions. When the operators are dual to strings at massive levels, then our methods are valid, since their dimensions scale as $2 \sqrt{n} \lambda^{1 / 4}$. However, we are forced to give large $R$-charges to protected operators, which makes it impossible to study three-point functions involving, say, the chiral primary in the 20. Another direction of study would be to compute subleading corrections to our results in the $\alpha^{\prime}$ expansion. Besides the corrections coming from the expansions of the dimensions, we would also need to calculate loop corrections to the worldsheet amplitude.

Another possibility is to study four-point correlators of short operators. In principle the main ideas behind our methods should still hold. When doing the saddle point approximation one would obtain two intersection points in the bulk, corresponding to the product of two three-point functions and exchange of some operator. It is possible that under some approximations this computation might become feasible. 
Perhaps yet another future application of our results involves the comparison to upper bounds of leading twist operators using bootstrap methods. In [46] the authors showed that the upper bounds are consistent with the supergravity correction in (3.16) for the $J=2$ double-trace state at large values of the central charge, $c=\frac{1}{4}\left(N^{2}-1\right)$. There is evidence that the upper bounds occur at special values of the coupling $[47,48]$, which corresponds to an 't Hooft coupling of order $\lambda \sim N$. For this value the splitting is large compared to the supergravity correction but also only affects double-traces with $J \sim N^{1 / 4}$ and so a present comparison cannot be made. If instead one could also expand the bootstrap analysis to include the 't Hooft parameter as an input, then one could in principle make such a comparison if at the same time one can find the splitting for low $J$ values.

Finally, a bigger goal is to understand the structure of the full $\mathrm{AdS}_{5} \times \mathrm{S}^{5}$ vertex operators and to use the underlying integrability of the string world-sheet to compute holographic three-point functions.

\section{Acknowledgments}

We thank T. Bargheer and V. Gonçalves for discussions. This research is supported in part by Vetenskapsrådet under grant \#2012-3269. JAM thanks the CTP at MIT for hospitality during the course of this work.

\section{A Vertex function contractions}

Two massless scalars and one Regge spin. When computing the three-point function of one Regge spin and two massless scalars, we need to compute only two amplitudes. The first, with three NS-NS vertices, is $\left\langle W_{1} W_{1} O_{n}\right\rangle$ which can be computed by using the result for the left movers (4.23) and taking $n_{1}=n_{2}=0$. Combining with the right mover part we have

$$
\left\langle W_{1} W_{1} O_{n}\right\rangle=-g_{\mathrm{c}}^{3}\left(\frac{\alpha^{\prime}}{2}\right)^{n-1} \epsilon_{M \tilde{M}}^{1} \epsilon_{N \tilde{N}}^{2}\left(\epsilon_{R S \tilde{R} \tilde{S}}^{3} \cdot k_{1}^{2 n-2}\right) X_{L}^{M N R S} X_{R}^{\tilde{M} \tilde{N} \tilde{R} \tilde{S}}
$$

with

$$
X_{L}^{M N R S}=n \eta^{M R} \eta^{N S}+\frac{\alpha^{\prime}}{2} k_{1}^{R}\left(k_{2}^{M} \eta^{N S}+k_{3}^{N} \eta^{M S}+k_{1}^{S} \eta^{M N}\right) .
$$

The other correlator is $\left\langle W_{2} W_{2} O_{n}\right\rangle$ which gives

$$
\left\langle W_{2} W_{2} O_{n}\right\rangle=-\frac{1}{2} g_{\mathrm{c}}^{3}\left(\frac{\alpha^{\prime}}{2}\right)^{n}\left(\epsilon_{M \tilde{M}}^{3} \cdot k_{1}^{2 n}\right) \operatorname{Tr}\left[t^{1} \Gamma^{M} C\left(t^{2}\right)^{\top} \Gamma^{\tilde{M}} C\right] .
$$

One massless scalar, one massive scalar and one Regge spin. To compute the three-point function of one massless scalar, one scalar at the first massive level and one Regge spin, we will need three amplitudes, two with three NS-NS vertices and one with two vertices in the R-R sector. Once again, for $\left\langle V_{1} W_{1} O_{n}\right\rangle$, we can use the left mover result (4.23) and take $n_{1}=1$ and $n_{2}=0$. Including also the right movers we obtain

$$
\left\langle V_{1} W_{1} O_{n}\right\rangle=-g_{\mathrm{c}}^{3}\left(\frac{\alpha^{\prime}}{2}\right)^{n-2} \epsilon_{M N \tilde{M} \tilde{N}}^{1} \epsilon_{P \tilde{P}}^{2}\left(\epsilon_{R S T \tilde{R} \tilde{S} \tilde{T}}^{3} \cdot k_{1}^{2 n-4}\right) X_{L}^{M N P R S T} X_{R}^{\tilde{M} \tilde{N} \tilde{P} \tilde{R} \tilde{S} \tilde{T}}
$$


with

$$
\begin{aligned}
X_{L}^{M N P R S T}= & \left(\frac{\alpha^{\prime}}{2}\right)^{2} k_{2}^{M} k_{1}^{R} k_{1}^{S}\left(k_{3}^{P} \eta^{N T}+k_{2}^{N} \eta^{P T}+k_{1}^{T} \eta^{N P}\right)+n(n-1) \eta^{M R} \eta^{N S} \eta^{P T} \\
& +\frac{\alpha^{\prime}}{2} k_{1}^{R} \eta^{M S}\left(n k_{3}^{P} \eta^{N T}+(1+n) k_{1}^{T} \eta^{N P}+2 n k_{2}^{N} \eta^{P T}\right) .
\end{aligned}
$$

We also need to compute $\left\langle V_{2} W_{1} O_{n}\right\rangle$ which is

$$
\left\langle V_{2} W_{1} O_{n}\right\rangle=-36 g_{\mathrm{c}}^{3}\left(\frac{\alpha^{\prime}}{2}\right)^{n+1} \alpha^{1{ }^{M N L}, \tilde{M} \tilde{N} \tilde{L}} k_{2 L} k_{2} \epsilon_{M \tilde{M}}^{2}\left(\epsilon_{N \tilde{N}}^{3} \cdot k_{1}^{2 n}\right) .
$$

The amplitude with $\mathrm{R}-\mathrm{R}$ vertices is

$$
\left\langle V_{3} W_{2} O_{n}\right\rangle=-\frac{1}{2} g_{\mathrm{c}}^{3}\left(\frac{\alpha^{\prime}}{2}\right)^{n-1}\left(\epsilon_{M N \tilde{M} \tilde{N}}^{3} \cdot k_{2}^{2 n-2}\right) \operatorname{Tr}\left[t^{2} X_{L}^{M N P}\left(t_{\tilde{P}, P}^{1}\right)^{\top}\left(X_{R}^{\tilde{M} \tilde{M} \tilde{P}}\right)^{\top}\right],
$$

where

$$
X_{L}^{M N P}=\left(n \eta^{M P}+\frac{\alpha^{\prime}}{2} k_{3}^{P} k_{2}^{M}\right)\left(\Gamma^{N} C\right)+\frac{\alpha^{\prime}}{2} k_{2}^{M} \eta^{N P}\left(\not k_{1} C\right) .
$$

Two massive scalars and one Regge spin. In this case we will need three amplitudes with three NS-NS vertices and one with two R-R vertices. For $\left\langle V_{1} V_{1} O_{n}\right\rangle$ we just take (4.23) with $n_{1}=n_{2}=1$, thus obtaining

$\left\langle V_{1} V_{1} O_{n}\right\rangle=-g_{\mathrm{c}}^{3}\left(\frac{\alpha^{\prime}}{2}\right)^{n-3} \epsilon_{M N \tilde{M} \tilde{N}}^{1} \epsilon_{P Q \tilde{P} \tilde{Q}}^{2}\left(\epsilon_{R S T V \tilde{R} \tilde{S} \tilde{T} \tilde{V}}^{3} \cdot k_{1}^{2 n-6}\right) X_{L}^{M N P Q R S T V} X_{R}^{\tilde{M} \tilde{N} \tilde{P} \tilde{Q} \tilde{R} \tilde{S} \tilde{T} \tilde{V}}$,

with

$$
\begin{aligned}
X_{L}^{M N} & \\
= & n \frac{\alpha^{\prime}}{2} k_{1}^{R} \eta^{M S} \eta^{P T}\left(2(n-1)\left(k_{3}^{Q} \eta^{N V}+k_{2}^{N} \eta^{Q V}\right)+(n+2) k_{1}^{V} \eta^{N Q}\right) \\
& +\left(\frac{\alpha^{\prime}}{2}\right)^{2} k_{1}^{R} k_{1}^{S}\left(n\left(k_{3}^{P} k_{3}^{Q} \eta^{M T} \eta^{N V}+k_{2}^{M} k_{2}^{N} \eta^{P T} \eta^{Q V}+3 k_{2}^{M} k_{3}^{P} \eta^{N T} \eta^{Q V}\right)\right. \\
& \left.+k_{1}^{T} \eta^{M P}\left(k_{1}^{V} \eta^{N Q}+(n+2)\left(k_{3}^{Q} \eta^{N V}+k_{2}^{N} \eta^{Q V}\right)\right)\right)+n(n-1)(n-2) \eta^{M R} \eta^{N S} \eta^{P T} \eta^{Q V} \\
& +\left(\frac{\alpha^{\prime}}{2}\right)^{3} k_{2}^{M} k_{3}^{P} k_{1}^{R} k_{1}^{S} k_{1}^{T}\left(k_{3}^{Q} \eta^{N V}+k_{2}^{N} \eta^{Q V}+k_{1}^{V} \eta^{N Q}\right) .
\end{aligned}
$$

The other correlators with NS-NS vertices are

$\left\langle V_{2} V_{2} O_{n}\right\rangle=-6^{2} g_{\mathrm{c}}^{3}\left(\frac{\alpha^{\prime}}{2}\right)^{n-1} \alpha_{M N L, \tilde{M} \tilde{N} \tilde{L}}^{1} \alpha_{O P Q, \tilde{O} \tilde{P} \tilde{Q}}^{2} \epsilon_{R S, \tilde{R} \tilde{S}}^{3} \cdot k_{1}^{2 n-2} X_{L}^{M N L O P Q R S} X_{R}^{\tilde{M} \tilde{N} \tilde{L} \tilde{O} \tilde{P} \tilde{R} \tilde{S}}$

with

$$
X_{L}^{M N L O P Q R S}=\eta^{N Q} \eta^{L P}\left(\frac{\alpha^{\prime}}{2} k_{1}^{R}\left(\eta^{M O} k_{1}^{S}+3\left(k_{3}^{O} \eta^{M S}+k_{2}^{M} \eta^{O S}\right)\right)+3 n \eta^{M R} \eta^{O S}\right),
$$

and

$$
\left\langle V_{2} V_{1} O_{n}\right\rangle=-36 g_{\mathrm{c}}^{3}\left(\frac{\alpha^{\prime}}{2}\right)^{n-1} \alpha_{M N L, \tilde{M} \tilde{N} \tilde{L}}^{1} \epsilon_{P Q, \tilde{P} \tilde{Q}}^{2}\left(\epsilon_{R S, \tilde{R} \tilde{S}}^{3} \cdot k_{1}^{2 n-2}\right) X_{L}^{M N L P Q R S} X_{R}^{\tilde{M} \tilde{N} \tilde{L} \tilde{P} \tilde{Q} \tilde{R} \tilde{S}},
$$


with

$$
X_{L}^{M N L P Q R S}=\sqrt{\frac{\alpha^{\prime}}{2}} \eta^{M R} \eta^{N P} k_{2}^{L}\left(n \eta^{Q S}+\frac{\alpha^{\prime}}{2} k_{3}^{Q} k_{1}^{S}\right) .
$$

The correlator with two R-R vertices is

$$
\left\langle V_{3} V_{3} O_{n}\right\rangle=-\frac{g_{\mathrm{c}}^{3}}{2}\left(\frac{\alpha^{\prime}}{2}\right)^{n-2}\left(\epsilon_{M N L \tilde{M} \tilde{N} \tilde{L}}^{3} \cdot k_{1}^{2 n-4}\right) \operatorname{Tr}\left[t_{\tilde{R}, R}^{1} X_{L}^{M N L R S}\left(t_{\tilde{S}, S}^{2}\right)^{\top}\left(X_{R}^{\tilde{M} \tilde{N} \tilde{L} \tilde{R} \tilde{S}}\right)^{\top}\right]
$$

where

$$
\begin{aligned}
& X_{L}^{M N L R S} \\
& =\left(n(n-1) \eta^{M R} \eta^{N S}+\frac{\alpha^{\prime}}{2} k_{1}^{M}\left(n\left(k_{3}^{S} \eta^{N R}+\eta^{N S} k_{2}^{R}\right)+k_{1}^{N} \eta^{R S}+\frac{\alpha^{\prime}}{2} k_{3}^{S} k_{1}^{N} k_{2}^{R}\right)\right)\left(\Gamma^{L} C\right) \\
& \quad+\frac{\alpha^{\prime}}{2} k_{1}^{M}\left(\eta^{N S}\left(n \eta^{L R}+\frac{\alpha^{\prime}}{2} k_{2}^{R} k_{1}^{L}\right)\left(k_{2} C\right)-\eta^{N R}\left(n \eta^{L S}+\frac{\alpha^{\prime}}{2} k_{3}^{S} k_{1}^{L}\right)\left(k_{1} C\right)+\frac{\alpha^{\prime}}{4} k_{1}^{N} \eta^{R S}\left(k_{1} \Gamma^{L} \not k_{2} C\right)\right) .
\end{aligned}
$$

One massless scalar and two Regge spins. When considering the correlator of two Regge spins with a massless scalar it is enough to use (4.23) with $n_{1}=0$ which, for $n_{2} \leq n_{3}$, gives for the left mover contractions

$$
\begin{aligned}
& \left\langle W_{1} O_{n_{2}} O_{n_{3}}\right\rangle_{L} \\
& =g_{\mathrm{c}}^{3} n_{2} ! n_{3} ! \sum_{k_{L}=0}^{s_{2}} \frac{\left(\alpha^{\prime} / 2\right)^{1 / 2\left(s_{2}+s_{3}-1\right)-k_{L}} \eta_{23}^{k_{L}}}{k_{L} !\left(s_{2}-k_{L}\right) !\left(s_{3}-k_{L}\right) !}\left[\frac{\alpha^{\prime}}{2} k_{L}\left(\epsilon^{1} \cdot k_{2}\right)\left(\epsilon^{2} \cdot k_{3}^{s_{2}-k_{L}}\right)\left(\epsilon^{3} \cdot k_{1}^{s_{3}-k_{L}}\right)\right. \\
& \left.\quad+s_{2}\left(s_{3}-k_{L}\right) \epsilon^{1^{M}}\left(\epsilon^{2} \cdot k_{3}^{s_{2}-k_{L}}\right)\left(\epsilon_{M}^{3} \cdot k_{1}^{s_{3}-k_{L}-1}\right)+s_{3}\left(s_{2}-k_{L}\right) \epsilon^{1^{M}}\left(\epsilon_{M}^{2} \cdot k_{3}^{s_{2}-k_{L}-1}\right)\left(\epsilon^{3} \cdot k_{1}^{s_{3}-k_{L}}\right)\right] .
\end{aligned}
$$

For the right movers we will have the same formula, with the sum running over $k_{R}$ instead. If we contract with the polarization of the dilaton and denote $k=k_{L}+k_{R}$ then we have

$$
\begin{aligned}
& \left\langle W_{1}^{\mathrm{T}} O_{S_{2}} O_{S_{3}}\right\rangle \\
& =g_{\mathrm{c}}^{3} \sum_{k=0}^{S_{2}} \sum_{l=\max \left(0, k-\frac{S_{2}}{2}\right)}^{\min \left(\frac{S_{2}}{2}, k\right)} \frac{\left(n_{2} ! n_{3} !\right)^{2}\left(\alpha^{\prime} / 2\right)^{S_{2} / 2+S_{3} / 2-k}\left(\epsilon_{M}^{2} \cdot k_{3}^{S_{2}-k-1}\right)\left(\epsilon_{N}^{3} \cdot k_{1}^{S_{3}-k-1}\right)}{l !\left(s_{2}-l\right) !\left(s_{3}-l\right) !(k-l) !\left(s_{2}-k+l\right) !\left(s_{3}-k+l\right) !} \\
& \quad \times \eta_{23}^{k}\left[\left(\frac{k S_{2} S_{3}}{4}-2 l(k-l)\right) k_{3}^{M} k_{1}^{N}+\frac{S_{2} S_{3}}{4 \alpha^{\prime}}\left(k\left(S_{2}+S_{3}\right)-S_{2} S_{3}-4 l(k-l)\right) \eta^{M N}\right] .
\end{aligned}
$$

If instead we contract with the polarization of the chiral operator then we obtain

$$
\begin{aligned}
& \left\langle W_{1} O_{S_{2}} O_{S_{3}}\right\rangle \\
& =g_{\mathrm{c}}^{3} \sum_{k=0}^{S_{2}} \sum_{l=\max \left(0, k-\frac{S_{2}}{2}\right)}^{\min \left(\frac{S_{2}}{2}, k\right)} \frac{\left(n_{2} ! n_{3} !\right)^{2}\left(\alpha^{\prime} / 2\right)^{S_{2} / 2+S_{3} / 2-k}\left(\epsilon_{M}^{2} \cdot k_{3}^{S_{2}-k-1}\right)\left(\epsilon_{N}^{3} \cdot k_{1}^{S_{3}-k-1}\right)}{l !\left(s_{2}-l\right) !\left(s_{3}-l\right) !(k-l) !\left(s_{2}-k+l\right) !\left(s_{3}-k+l\right) !} \\
& \quad \times \eta_{23}^{k}\left[\left(\frac{-k S_{2} S_{3}}{4}+(2+\delta) l(k-l)\right) k_{3}^{M} k_{1}^{N}-\frac{S_{2} S_{3}}{4 \alpha^{\prime}}\left(k\left(S_{2}+S_{3}\right)-S_{2} S_{3}-4 l(k-l)\right) \eta^{M N}\right],
\end{aligned}
$$

with

$$
\delta=\frac{1}{\sqrt{\lambda} \Delta_{1}^{2}}\left(\sqrt{\lambda}\left(\left(S_{2}+S_{3}-4\right) \Delta_{1}^{2}-\left(S_{2}-S_{3}\right)\left(\Delta_{2}^{2}-\Delta_{3}^{2}\right)\right)+\lambda\left(S_{2}-S_{3}\right)^{2}-4 \alpha_{1} \alpha_{2} \alpha_{3} \Sigma\right) .
$$


One massive scalar and two Regge spins. For this correlator there are two amplitudes we must consider. The first one can be taken by using (4.23) with $n_{1}=1$ which, for $n_{2} \leq n_{3}$, gives for the left mover contractions

$$
\begin{aligned}
\left\langle V_{1} O_{n_{2}} O_{n_{3}}\right\rangle_{L} & \\
= & g_{\mathrm{c}}^{3} n_{2} ! n_{3} ! \sum_{k_{L}=0}^{s_{2}} \frac{\left(\alpha^{\prime} / 2\right)^{1 / 2\left(s_{2}+s_{3}\right)-k_{L}-1} \eta_{23}^{k_{L}}}{k_{L} !\left(s_{2}-k_{L}\right) !\left(s_{3}-k_{L}\right) !} \epsilon_{M N}^{1}\left(\epsilon_{P Q}^{2} \cdot k_{3}^{s_{2}-k_{L}-2}\right)\left(\epsilon_{R S}^{3} \cdot k_{1}^{s_{3}-k_{L}-2}\right) \\
& \times\left[s_{3}\left(s_{2}-k_{L}\right)\left(n_{2}-k_{L}\right) \eta^{M P} \eta^{N Q} k_{1}^{R} k_{1}^{S}+s_{2}\left(s_{3}-k_{L}\right)\left(n_{3}-k_{L}\right) \eta^{M R} \eta^{N S} k_{3}^{P} k_{3}^{Q}\right. \\
& +\left(s_{2}+s_{3}-1\right)\left(s_{2}-k_{L}\right)\left(s_{3}-k_{L}\right) \eta^{M P} \eta^{N R} k_{3}^{Q} k_{1}^{S}+\left(\frac{\alpha^{\prime}}{2}\right)^{2} k_{L} k_{2}^{M} k_{2}^{N} k_{3}^{P} k_{3}^{Q} k_{1}^{R} k_{1}^{S} \\
& \left.+\frac{\alpha^{\prime}}{2} k_{2}^{M} k_{3}^{P} k_{1}^{R}\left(\left(s_{3}+k_{L}\right)\left(s_{2}-k_{L}\right) \eta^{N Q} k_{1}^{S}+\left(s_{2}+k_{L}\right)\left(s_{3}-k_{L}\right) \eta^{N S} k_{3}^{Q}\right)\right] .
\end{aligned}
$$

For the right movers we will have an analogous formula, so contracting with the polarization of the Konishi opeartor and using $k=k_{L}+k_{R}$ leads to

$$
\begin{aligned}
& \left\langle V_{1} O_{n_{2}} O_{n_{3}}\right\rangle \\
& =g_{\mathrm{c}}^{3} \sum_{k=0}^{S_{2}} \sum_{l=\max \left(0, k-\frac{S_{2}}{2}\right)}^{\min \left(\frac{S_{2}}{2}, k\right)} \frac{\left(n_{2} ! n_{3} !\right)^{2}\left(\alpha^{\prime} / 2\right)^{\frac{S_{2}+S_{3}}{2}}-k\left(\epsilon_{M N}^{2} \cdot k_{3}^{S_{2}-k-2}\right)\left(\epsilon_{P Q}^{3} \cdot k_{1}^{S_{3}-k-2}\right) \eta_{23}^{k}}{l !\left(s_{2}-l\right) !\left(s_{3}-l\right) !(k-l) !\left(s_{2}-k+l\right) !\left(s_{3}-k+l\right) !} \\
& \times\left[K_{1}\left(k, l, S_{i}, \Delta_{i}\right) k_{3}^{M} k_{3}^{N} k_{1}^{P} k_{1}^{Q}+\frac{2}{\alpha^{\prime}} K_{2}\left(k, l, S_{i}, \Delta_{i}\right) \eta^{M P} k_{3}^{N} k_{1}^{Q}+\frac{4}{\alpha^{\prime 2}} K_{3}\left(k, l, S_{i}, \Delta_{i}\right) \eta^{M P} \eta^{N Q}\right],
\end{aligned}
$$

with

$$
\begin{aligned}
K_{1} & \left(k, l, S_{i}, \Delta_{i}\right) \\
= & \frac{k S}{32 \lambda}\left(\Delta_{-}^{2}-4 \sqrt{\lambda}\left(\Delta_{+}+S_{-} \Delta_{-}\right)\right)-\frac{l(k-l)}{256 \lambda^{2}} \Delta_{-}^{2}\left(\Delta_{-}^{2}-8 \sqrt{\lambda}\left(2 \Delta_{+}+S_{-} \Delta_{-}\right)\right) \\
& +\frac{S}{96}\left(4 S_{+} k\left(3 S_{+}+2\right)-\left(3 S+28 k+48 S k-12 k^{2}\right)\right)-\frac{l(k-l)}{288 \lambda}(144 \lambda l(k-l) \\
& -36 \sqrt{\lambda}\left(\left(S_{-}\left(S_{-}^{2}+2 S_{+}+4 k\right)+4 S_{-}\right) \Delta_{-}+2\left(2 k-6+2 S_{+}+S_{-}^{2}\right) \Delta_{+}\right) \\
& +9\left(\left(3 S_{-}^{2}+2\left(S_{+}+2 k+1\right)\right) \Delta_{-}^{2}+8 S_{-} \Delta_{-} \Delta_{+}-16 \Delta_{2}^{2} \Delta_{3}^{2}\right)+2 \lambda\left(9 S_{+}^{4}+36 S_{+}^{3}\right. \\
& \left.\left.-2(11+36(S-k)) S_{+}^{2}-8(13+18 S-15 k) S_{+}+4(56+9 S(3+4 S-8 k))\right)\right), \\
K_{2} & \left(k, l, S_{i}, \Delta_{i}\right) \\
= & \frac{l(k-l)}{144}\left(9 S_{+}^{4}+22 S_{+}^{3}+2 S_{+}^{2}(13 k-45 S-32)+4 S_{+}(10-27 S-8 k)\right. \\
& \left.+8(27 S(S+1)-5 k)-12 l(k-l)\left(3 S_{-}^{2}+16\left(S_{+}-2\right)\right)\right) \\
& -\frac{S_{2} S_{3}}{576}\left(S_{+}^{2}\left(9 S+34 k-36 k^{2}\right)-4 S_{+}\left(3 S+22 k+18 k^{2}\right)+8\left(3 S-4 S^{2}+5 k+18 k^{2}(1+S)\right)\right) \\
& -\frac{1}{256 \lambda}\left(S\left(S-4 k^{2}\right)+4 l(k-l)\left(4 l(k-l)-\left(S_{-}^{2}-2 S\right)\right)\right)\left(\Delta_{-}^{2}-4 \sqrt{\lambda}\left(2 \Delta_{+}+S_{-} \Delta_{-}\right)\right),
\end{aligned}
$$




$$
\begin{aligned}
& K_{3}\left(k, l, S_{i}, \Delta_{i}\right) \\
& =\frac{S}{1152}\left(4 S_{+}\left(25 S+50 k+18 k S+64 k^{2}\right)-S_{+}^{2}\left(7 S+128 k-100 k^{2}\right)\right. \\
& \left.\quad+14 k S_{+}^{3}-2\left(18 S^{2}+86 S+72 S k+57 k^{2}-72 S k^{2}\right)\right) \\
& \quad+\frac{l(k-l)}{288}\left(-7 S_{+}^{4}+14(k+2) S_{+}^{3}+\left(50 S_{2} S_{3}-56 k-28\right) S_{+}^{2}+8(S(9 k+2)+7 k) S_{+}\right. \\
& \left.\quad-8\left(27 S^{2}+18 k S+11\right)-4 l(k-l)\left(7\left(S_{+}-2\right)^{2}+36 S\right)\right),
\end{aligned}
$$

where we defined $S_{-}=S_{2}-S_{3}, S_{+}=S_{2}+S_{3}, S=S_{2} S_{3}, \Delta_{-}=\Delta_{2}^{2}-\Delta_{3}^{2}$ and $\Delta_{+}=\Delta_{2}^{2}+\Delta_{3}^{2}$. The other correlator needed is, for $n_{2} \leq n_{3}$

$$
\left\langle V_{2} O_{n_{2}} O_{n_{3}}\right\rangle_{L}=g_{\mathrm{c}}^{3} n_{2} ! n_{3} ! \sum_{k_{L}=0}^{n 2} \frac{6\left(\alpha^{\prime} / 2\right)^{-k_{L}+\frac{n_{2}+n_{3}+1}{2}} \eta_{23}^{k_{L}}}{k_{L} !\left(n_{2}-k_{L}\right) !\left(n_{3}-k_{L}\right) !} \alpha_{1}^{M N L} \epsilon_{M}^{2} k_{3}^{n_{2}-k_{L}} \epsilon_{N}^{3} k_{1}^{n_{3}-k_{L}} k_{3 L} .
$$

Combining with the contractions from the right movers and contracting indices from the scalar polarization, we get for $k=k_{L}+k_{R}$

$$
\begin{aligned}
& \left\langle V_{2} O_{S_{2}} O_{S_{3}}\right\rangle \\
& =g_{\mathrm{c}}^{3} \sum_{k=0}^{S_{2}} \sum_{l=\max \left(0, k-\frac{S_{2}}{2}\right)}^{\min \left(\frac{S_{2}}{2}, k\right)} \frac{\left(n_{2} ! n_{3} !\right)^{2}\left(\alpha^{\prime} / 2\right)^{\frac{S_{2}+S_{3}}{2}-k}\left(\epsilon_{M N}^{2} \cdot k_{3}^{S_{2}-k-2}\right)\left(\epsilon_{P Q}^{3} \cdot k_{1}^{S_{3}-k-2}\right) \eta_{23}^{k}}{l !\left(n_{2}-l\right) !\left(n_{3}-l\right) !(k-l) !\left(n_{2}-k+l\right) !\left(n_{3}-k+l\right) !} \\
& \quad \times\left[-\frac{1}{2} k_{3}^{M} k_{3}^{N} k_{1}^{P} k_{1}^{Q}+\frac{2}{\alpha^{\prime}} K_{4}\left(S_{i}, \Delta_{i}\right) \eta^{M P} k_{3}^{N} k_{1}^{Q}+\frac{4}{\alpha^{\prime 2}} K_{5}\left(S_{i}, \Delta_{i}\right) \eta^{M P} \eta^{N Q}\right],
\end{aligned}
$$

where

$$
\begin{aligned}
& K_{4}\left(S_{i}, \Delta_{i}\right)=-\frac{1}{16 \lambda}\left(4 \lambda\left(S_{-}^{2}-2 S_{+}+4\right)+\Delta_{-}^{2}+4 \sqrt{\lambda}\left(2 \Delta_{+}-S_{-} \Delta_{-}\right)\right), \\
& K_{5}\left(S_{i}, \Delta_{i}\right)=\frac{1}{16 \lambda}\left(2\left(S_{-}^{2}-4 S_{+}+12\right) \lambda-\Delta_{-}^{2}+8 \sqrt{\lambda} \Delta_{+}\right) .
\end{aligned}
$$

Putting the two correlators together we get

$$
\left\langle V_{K} V_{S_{2}} V_{S_{3}}\right\rangle=\sum_{k=0}^{S_{2}}\left(\alpha^{\prime} / 2\right)^{S_{2} / 2+S_{3} / 2-k}\left(\epsilon^{2} \cdot k_{3}^{S_{2}-k}\right)\left(\epsilon^{3} \cdot k_{1}^{S_{3}-k}\right) \eta_{23}^{k} \mathcal{K}_{k},
$$

with the coefficients $\mathcal{K}_{k}$ given by

$$
\begin{aligned}
\mathcal{K}_{k}= & g_{\mathrm{c}}^{3} \sum_{l=\max \left(0, k-\frac{S_{2}}{2}\right)}^{\min \left(\frac{S_{2}}{2}, k\right)} \frac{n_{2} ! n_{3} !}{16 l !\left(s_{2}-l\right) !\left(s_{3}-l\right) !(k-l) !\left(s_{2}-k+l\right) !\left(s_{3}-k+l\right) !} \times \\
\times & {\left[K_{1}\left(k, l, S_{i}, \Delta_{i}\right)+\frac{(k-l)}{\left(s_{2}-k+l+1\right)\left(s_{3}-k+l+1\right)} K_{2}\left(k-1, l, S_{i}, \Delta_{i}\right)\right.} \\
& +\frac{(k-l)(k-l-1)}{\left(s_{2}-k+l+1\right)\left(s_{3}-k+l+1\right)\left(s_{2}-k+l+2\right)\left(s_{3}-k+l+2\right.} K_{3}\left(k-2, l, S_{i}, \Delta_{i}\right) \\
& -\frac{\left(s_{2}-k+l\right)\left(s_{3}-k+l\right)\left(s_{2}-l\right)\left(s_{3}-l\right)}{2}+(k-l)\left(s_{2}-l\right)\left(s_{3}-l\right) K_{4}\left(S_{i}, \Delta_{i}\right) \\
& \left.+\frac{(k-l)(k-l-1)\left(s_{2}-l\right)\left(s_{3}-l\right)}{\left(s_{2}-k+l+1\right)\left(s_{3}-k+l+1\right)} K_{5}\left(S_{i}, \Delta_{i}\right)\right] .
\end{aligned}
$$


Open Access. This article is distributed under the terms of the Creative Commons Attribution License (CC-BY 4.0), which permits any use, distribution and reproduction in any medium, provided the original author(s) and source are credited.

\section{References}

[1] N. Beisert et al., Review of AdS/CFT Integrability: An Overview, Lett. Math. Phys. 99 (2012) 3 [arXiv: 1012.3982] [INSPIRE].

[2] R. Roiban and A. Volovich, Yang-Mills correlation functions from integrable spin chains, JHEP 09 (2004) 032 [hep-th/0407140] [INSPIRE].

[3] L.F. Alday, J.R. David, E. Gava and K.S. Narain, Structure constants of planar $N=4$ Yang-Mills at one loop, JHEP 09 (2005) 070 [hep-th/0502186] [INSPIRE].

[4] L.F. Alday, J.R. David, E. Gava and K.S. Narain, Towards a string bit formulation of $N=4$ super Yang-Mills, JHEP 04 (2006) 014 [hep-th/0510264] [INSPIRE].

[5] J. Escobedo, N. Gromov, A. Sever and P. Vieira, Tailoring Three-Point Functions and Integrability, JHEP 09 (2011) 028 [arXiv:1012.2475] [INSPIRE].

[6] J. Escobedo, N. Gromov, A. Sever and P. Vieira, Tailoring Three-Point Functions and Integrability II. Weak/strong coupling match, JHEP 09 (2011) 029 [arXiv:1104.5501] [INSPIRE].

[7] N. Gromov, A. Sever and P. Vieira, Tailoring Three-Point Functions and Integrability III. Classical Tunneling, JHEP 07 (2012) 044 [arXiv:1111.2349] [INSPIRE].

[8] A. Bissi, T. Harmark and M. Orselli, Holographic 3-Point Function at One Loop, JHEP 02 (2012) 133 [arXiv:1112.5075] [INSPIRE].

[9] I. Kostov, Three-point function of semiclassical states at weak coupling, J. Phys. A 45 (2012) 494018 [arXiv: 1205 .4412] [INSPIRE].

[10] V. Kazakov and E. Sobko, Three-point correlators of twist-2 operators in N $=4 S Y M$ at Born approximation, JHEP 06 (2013) 061 [arXiv:1212.6563] [INSPIRE].

[11] N. Gromov and P. Vieira, Tailoring Three-Point Functions and Integrability IV. Theta-morphism, JHEP 04 (2014) 068 [arXiv:1205.5288] [INSPIRE].

[12] O. Foda, Y. Jiang, I. Kostov and D. Serban, A tree-level 3-point function in the SU(3)-sector of planar $N=4$ SYM, JHEP 10 (2013) 138 [arXiv:1302.3539] [INSPIRE].

[13] P. Vieira and T. Wang, Tailoring Non-Compact Spin Chains, JHEP 1410 (2014) 35 [arXiv: 1311.6404] [INSPIRE].

[14] J. Caetano and T. Fleury, Three-point functions and $\mathfrak{s u}(1 \mid 1)$ spin chains, JHEP 09 (2014) 173 [arXiv: 1404.4128] [INSPIRE].

[15] Y. Jiang, I. Kostov, F. Loebbert and D. Serban, Fixing the Quantum Three-Point Function, JHEP 04 (2014) 019 [arXiv: 1401.0384] [INSPIRE].

[16] K. Zarembo, Holographic three-point functions of semiclassical states, JHEP 09 (2010) 030 [arXiv: 1008.1059] [INSPIRE].

[17] M.S. Costa, R. Monteiro, J.E. Santos and D. Zoakos, On three-point correlation functions in the gauge/gravity duality, JHEP 11 (2010) 141 [arXiv:1008.1070] [INSPIRE]. 
[18] R.A. Janik, P. Surowka and A. Wereszczynski, On correlation functions of operators dual to classical spinning string states, JHEP 05 (2010) 030 [arXiv:1002.4613] [INSPIRE].

[19] E.I. Buchbinder and A.A. Tseytlin, Semiclassical correlators of three states with large $S^{5}$ charges in string theory in $A d S_{5} \times S^{5}$, Phys. Rev. D 85 (2012) 026001 [arXiv:1110.5621] [INSPIRE].

[20] A. Bissi, C. Kristjansen, D. Young and K. Zoubos, Holographic three-point functions of giant gravitons, JHEP 06 (2011) 085 [arXiv:1103.4079] [INSPIRE].

[21] G. Georgiou, SL(2) sector: weak/strong coupling agreement of three-point correlators, JHEP 09 (2011) 132 [arXiv: 1107.1850] [INSPIRE].

[22] R.A. Janik and A. Wereszczynski, Correlation functions of three heavy operators: The AdS contribution, JHEP 12 (2011) 095 [arXiv:1109.6262] [INSPIRE].

[23] Y. Kazama and S. Komatsu, On holographic three point functions for GKP strings from integrability, JHEP 01 (2012) 110 [arXiv:1110.3949] [INSPIRE].

[24] Y. Kazama and S. Komatsu, Wave functions and correlation functions for GKP strings from integrability, JHEP 09 (2012) 022 [arXiv:1205.6060] [INSPIRE].

[25] Y. Kazama and S. Komatsu, Three-point functions in the SU(2) sector at strong coupling, JHEP 03 (2014) 052 [arXiv: 1312.3727] [INSPIRE].

[26] L.F. Alday and A. Bissi, Higher-spin correlators, JHEP 10 (2013) 202 [arXiv:1305.4604] [INSPIRE].

[27] Z. Bajnok, R.A. Janik and A. Wereszczyński, HHL correlators, orbit averaging and form factors, JHEP 09 (2014) 050 [arXiv: 1404.4556] [INSPIRE].

[28] J.A. Minahan, Holographic three-point functions for short operators, JHEP 07 (2012) 187 [arXiv: 1206.3129] [INSPIRE].

[29] T. Bargheer, J.A. Minahan and R. Pereira, Computing Three-Point Functions for Short Operators, JHEP 03 (2014) 096 [arXiv:1311.7461] [INSPIRE].

[30] O. Schlotterer, Higher Spin Scattering in Superstring Theory, Nucl. Phys. B 849 (2011) 433 [arXiv: 1011.1235] [INSPIRE].

[31] M.S. Costa, V. Goncalves and J. Penedones, Conformal Regge theory, JHEP 12 (2012) 091 [arXiv: 1209.4355] [INSPIRE].

[32] E. Witten, Anti-de Sitter space and holography, Adv. Theor. Math. Phys. 2 (1998) 253 [hep-th/9802150] [INSPIRE].

[33] D.Z. Freedman, S.D. Mathur, A. Matusis and L. Rastelli, Correlation functions in the CFT $(d) / A d S(d+1)$ correspondence, Nucl. Phys. B 546 (1999) 96 [hep-th/9804058] [INSPIRE].

[34] S. Lee, S. Minwalla, M. Rangamani and N. Seiberg, Three point functions of chiral operators in $D=4, N=4 S Y M$ at large- $N$, Adv. Theor. Math. Phys. 2 (1998) 697 [hep-th/9806074] [INSPIRE].

[35] E. D'Hoker, D.Z. Freedman, S.D. Mathur, A. Matusis and L. Rastelli, Extremal correlators in the AdS/CFT correspondence, hep-th/9908160 [INSPIRE].

[36] T. Klose and T. McLoughlin, A light-cone approach to three-point functions in $A d S_{5} \times S^{5}$, JHEP 04 (2012) 080 [arXiv: 1106.0495] [INSPIRE]. 
[37] F.A. Dolan and H. Osborn, Superconformal symmetry, correlation functions and the operator product expansion, Nucl. Phys. B 629 (2002) 3 [hep-th/0112251] [INSPIRE].

[38] N. Beisert, BMN operators and superconformal symmetry, Nucl. Phys. B 659 (2003) 79 [hep-th/0211032] [INSPIRE].

[39] J.A. Minahan and K. Zarembo, The Bethe ansatz for $\mathcal{N}=4$ super Yang-Mills, JHEP 03 (2003) 013 [hep-th/0212208] [INSPIRE].

[40] G. Mack, Convergence of operator product expansions on the vacuum in conformal invariant quantum field theory, Commun. Math. Phys. 53 (1977) 155.

[41] G. Sotkov and R. Zaikov, Conformal invariant two and three-point functions for fields with arbitrary spin, Rept. Math. Phys. 12 (1977) 375.

[42] H. Osborn and A.C. Petkou, Implications of conformal invariance in field theories for general dimensions, Annals Phys. 231 (1994) 311 [hep-th/9307010] [INSPIRE].

[43] M.S. Costa, J. Penedones, D. Poland and S. Rychkov, Spinning Conformal Correlators, JHEP 11 (2011) 071 [arXiv:1107.3554] [INSPIRE].

[44] A. Sagnotti and M. Taronna, String Lessons for Higher-Spin Interactions, Nucl. Phys. B 842 (2011) 299 [arXiv: 1006.5242] [INSPIRE].

[45] G. Arutyunov and S. Frolov, Some cubic couplings in type IIB supergravity on $A d S_{5} \times S^{5}$ and three point functions in SYM(4) at large-N, Phys. Rev. D 61 (2000) 064009 [hep-th/9907085] [INSPIRE].

[46] C. Beem, L. Rastelli and B.C. van Rees, The $\mathcal{N}=4$ Superconformal Bootstrap, Phys. Rev. Lett. 111 (2013) 071601 [arXiv: 1304.1803] [INSPIRE].

[47] C. Beem, L. Rastelli, A. Sen and B.C. van Rees, Resummation and S-duality in $\mathcal{N}=4$ SYM, JHEP 04 (2014) 122 [arXiv: 1306.3228] [INSPIRE].

[48] L.F. Alday and A. Bissi, Modular interpolating functions for $N=4$ SYM, JHEP 07 (2014) 007 [arXiv: 1311.3215] [INSPIRE]. 